Research Article

\title{
Classical and Bayesian Estimation of the Inverse Weibull Distribution: Using Progressive Type-I Censoring Scheme
}

\author{
Ali Algarni, ${ }^{1}$ Mohammed Elgarhy $\mathbb{D}^{\circ},{ }^{2}$ Abdullah M Almarashi, ${ }^{1}$ Aisha Fayomi, ${ }^{1}$ \\ and Ahmed R El-Saeed ${ }^{3}$ \\ ${ }^{1}$ Statistics Department, Faculty of Science, King AbdulAziz University, Jeddah 21 551, Saudi Arabia \\ ${ }^{2}$ The Higher Institute of Commercial Sciences, Al Mahalla Al Kubra, Algarbia 31 951, Egypt \\ ${ }^{3}$ Department of Basic Sciences, Obour High Institute for Management \& Informatics, Cairo, Egypt
}

Correspondence should be addressed to Mohammed Elgarhy; m_elgarhy85@sva.edu.eg

Received 4 October 2021; Revised 4 November 2021; Accepted 20 November 2021; Published 29 December 2021

Academic Editor: AFAQ AHMAD

Copyright ( $\odot 2021$ Ali Algarni et al. This is an open access article distributed under the Creative Commons Attribution License, which permits unrestricted use, distribution, and reproduction in any medium, provided the original work is properly cited.

The challenge of estimating the parameters for the inverse Weibull (IW) distribution employing progressive censoring TypeI (PCTI) will be addressed in this study using Bayesian and non-Bayesian procedures. To address the issue of censoring time selection, qauntiles from the IW lifetime distribution will be implemented as censoring time points for PCTI. Focusing on the censoring schemes, maximum likelihood estimators (MLEs) and asymptotic confidence intervals (ACI) for unknown parameters are constructed. Under the squared error (SEr) loss function, Bayes estimates (BEs) and concomitant maximum posterior density credible interval estimations are also produced. The BEs are assessed using two methods: Lindley's approximation ( $\mathrm{LiA})$ technique and the Metropolis-Hasting $(\mathrm{MH})$ algorithm utilizing Markov Chain Monte Carlo (MCMC). The theoretical implications of MLEs and BEs for specified schemes of PCTI samples are shown via a simulation study to compare the performance of the different suggested estimators. Finally, application of two real data sets will be employed.

\section{Introduction}

Keller and Kamath [1] were the ones to propose the IW model as a sustainable idea for describing the deterioration of structural devices in diesel engines. The IW distribution gives an excellent match to various real data sets, according to [2]. In the perspective of a mechanical system's load-strength relationship, Calabria and Pulcini [3] gave an essential explanation of this distribution. The IW distribution, which was created to explain failures of structural devices influenced by degradation phenomena, plays a critical part in reliability engineering and lifetime testing. It has been looked into from a variety of angles. On the basis of the PC type-II data set, Musleh and Helu [4] used both conventional and Bayesian estimation techniques to estimate parameters from the IW distribution. Singh et al. [5] evaluated simulated hazards of several estimators, with a focus on the Bayesian approach. De Gusmao et al. [6] and Elbatal and Muhammed [7] focused their efforts on its comprehensive versions research, which included both generalized and exponentiated generalized IW distributions.

The distribution of IW has been studied from many angles. Khan et al. [8] visually and quantitatively depicted several aspects of this distribution, including mean $(\mathrm{M})$, variance $(V)$, kurtosis, and skewness. Erto [9] calculated the IW distribution using a fresh prior distribution, taking into account the shape parameter's range and the predicted value of a quantile $(Q)$ of the sampling distribution. Sultan et al. [10] go into great depth on how to include two IW distributions into a hybrid model. The IW distribution's density function (pdf), cumulative function (cdf), and $Q$ function $(Q f)$ are shown as follows: 


$$
\begin{aligned}
& f(x)=\gamma \lambda x^{-(\gamma+1)} \exp \left(\frac{-\lambda}{x^{\gamma}}\right), x>0, \gamma, \lambda>0, \\
& F(x)=\exp \left(\frac{-\lambda}{x^{\gamma}}\right), x>0, \gamma, \lambda>0, \\
& Q(u)=\sqrt[\gamma]{\frac{\lambda}{\ln (1 / u)}} .
\end{aligned}
$$

Note that when $\gamma=1$ and $\gamma=2$ the IW model reduces to inverted exponential (IE) inverted Rayleigh (IR) models.

Censored data arises in real-life testing trials when the experiments, which include the lifetime of test units, must be stopped before acquiring complete observation. For a variety of reasons, including time constraints and cost minimization, the censoring method is frequent and inescapable in practice. Various types of censorship have been explored in depth, with Type-I and Type-II censoring being the most prevalent. In comparison to classic censoring designs, a generalized type of censoring known as PC schemes has recently garnered substantial attention in the works as a result of its efficient use of available resources. PCTI is one of these PC schemes. When a certain number of lifetime test units are continually eliminated from the test at the conclusion of each of the post periods of time, this pattern is seen [11].

Assume there are $n$ units in a life testing experiment. Assume that $X_{1}, X_{2}, \ldots, X_{n}$ indicate the lifetime of all these $n$ units drawn from a population. The equivalent ordered lifetimes recorded from the life test are denoted by $x_{(1)}<x_{(2)}<\cdots<x_{(n)}$. Eventually $R_{i}$ items are omitted from the surviving items at the predetermined period of censoring $T_{q_{i}}$ in accordance with $q_{i}^{\text {th }}$ Qs, $i=1,2, \ldots, m$, where $m$ denotes the number of testing stages, $T_{q_{i}}>T_{q_{i-1}}$ and $n=r+\sum_{i=1}^{m} R_{i}$. The values $T_{q_{i}}$ must be established beforehand:

(1) According on the experimenter's prior knowledge and expertise with the items on test [12].

(2) The $Q s$ of lifetimes distribution, $q_{i}$ th, which may be constructed using the given formula

$$
P\left(X \leq T_{q_{i}}\right)=q_{i} \Rightarrow T_{q_{i}}=F^{-1}\left(q_{i}\right) i=1,2, \ldots, m .
$$

In these situations, $R_{i}, T_{q_{i}}$, and $n$ are preset and constant, whereas $l_{i}$ is the number of surviving objects at a given point in time $T_{q_{i}}$ and $r=\sum_{i=1}^{m} l_{i}$ are random variables. The LL function is indicated by

$$
\ell(\theta) \propto \prod_{i=1}^{r} f\left(x_{(i)} ; \theta\right) \prod_{j=1}^{m}\left(1-F\left(T_{\left(q_{j}\right)} ; \theta\right)\right)^{R_{j}}
$$

where $x_{(i)}$ is the observed lifetime of the $i$ th order statistic [13]. Figure 1 describes this scheme of censoring [14].

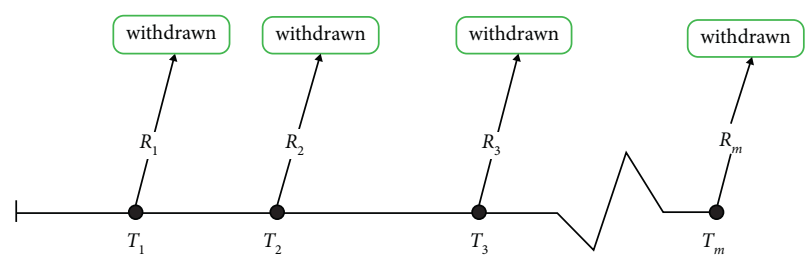

Figure 1: PCTI scheme.

One can observe that complete samples and also Type-I censoring scheme can be considered as special cases of this scheme of censoring.

Cohen [13] introduced PCTI scheme for the Weibull distribution. Mahmoud et al. [15] derived the MLLEs and the BEs for the parameters of the generalized IE model under PCTI. There are two closely related papers for the PCTI. The first one was the MLEs and ACI estimates for the unknown parameters of the generalized IE model under the idea that there are two types of failures [16]. The MLLEs and BEs for the unknown parameters of the generalized IE model [17] were the second.

The purpose of this paper is to look at the PCTI scheme when the lifetimes have their own IW model. We use two distinct techniques to drive the MLEs and BEs and derive the ACI of these different parameters: MCMC and Lindley Approximation. We look at a simulation outcome and a real data set to see how the various models perform in practice. The following is how the remaining of the article is structured: The MLLE and confidence intervals are discussed in Section 2. In Section 3, the Metropolis-Hasting (MH) algorithm and LiA are used to explore the Bayesian estimation technique, fully accrediting the gamma distribution as a prior distribution for unknown parameters. A simulated outcome and a real data set are utilized to demonstrate the theoretical conclusions in Section 5. Finally, there are some final observations and a summary.

\section{Estimation Using Method of Maximum Likelihood}

According on the PCTI method, for the unknown parameters of the IW distribution, the MLLE technique of estimate is examined in this section. This is how the PCTI system can be put into practice:

(i) Suppose a random sample of $n$ units with the next lifetime IW $(\gamma, \lambda)$ distribution to the test in a reallife experiment.

(ii) Prefix $m$ censoring time points $T_{q_{1}}, \ldots, T_{q_{m}}$, at which fixed number $R_{1}, \ldots, \ldots, R_{m-1}$ of surviving items are randomly omitted from the test. The censoring times $T_{q_{j}}$ are chosen corresponding to $P\left(X \leq T_{q_{j}}\right)=q_{j}$, where $X$ follows IW $(\gamma, \lambda)$ distribution and $q_{j}^{t h}$ is the Qs $(j=1,2, \ldots, m)$ of the chosen lifetime distribution.

(iii) The life test terminates at or before a prespecified time $T_{q_{m}}$. 
Therefore, one can obtain PCTI samples $x=\left(x_{(1)}, x_{(2)}, \ldots, x_{(r)}\right)$ that indicate the reported lifetime of $r$ units under that same censoring procedure.

By applying equation (1) of IW distribution in equation (4) of LL function under PCTI, the connected LL function of $\gamma$ and $\lambda$ given the PCTI data, $x$, may be interpreted as

$$
\ell(\gamma, \lambda) \propto(\gamma \lambda)^{r} \prod_{i=1}^{r}\left(x_{i}^{-(\gamma+1)} \exp \left(\frac{-\lambda}{x_{i}^{\gamma}}\right)\right) \prod_{j=1}^{m}\left(1-\exp \left(\frac{-\lambda}{T_{q_{j}}^{\gamma}}\right)\right)^{R_{j}} .
$$

Take logarithm of $\ell(\gamma, \lambda)$ to obtain $\log$-LL $\mathscr{L}$ as

$$
\begin{aligned}
\mathscr{L} \propto r \ln \gamma+r \ln \lambda-(\gamma+1) \sum_{i=1}^{r} \ln x_{i}-\lambda \sum_{i=1}^{r} x_{i}^{-\gamma} \\
\quad+\sum_{j=1}^{m} R_{j} \ln \left[1-\exp \left(\frac{-\lambda}{T_{q_{j}}^{\gamma}}\right)\right] .
\end{aligned}
$$

First partial derivatives of log-LL function $\mathscr{L}$ in terms of $\gamma$ and $\lambda$ are computed as follows:

$$
\begin{aligned}
& \frac{\partial \mathscr{L}}{\partial \gamma}=\frac{r}{\gamma}-\sum_{i=1}^{n} \ln x_{i}+\lambda \sum_{i=1}^{r} x_{i}^{-\gamma} \ln x_{i}-\sum_{j=1}^{m} \frac{\lambda R_{j} \ln T_{q_{j}}}{A_{j}}, \\
& \frac{\partial \mathscr{L}}{\partial \lambda}=\frac{r}{\lambda}-\sum_{i=1}^{r} x_{i}^{-\gamma}+\sum_{j=1}^{m} \frac{R_{j}}{A_{j}},
\end{aligned}
$$

where $A_{j}=T_{q_{j}}^{\gamma}\left[\exp \left(\lambda / T_{q_{j}}^{\gamma}\right)-1\right]$.

Equating $\left.(\partial \mathscr{L} / \partial \gamma)\right|_{\gamma=\hat{\gamma}}$ and $\left.(\partial \mathscr{L} / \partial \lambda)\right|_{\lambda=\hat{\lambda}}$ to 0 , then the numerical solution of the above two equations for $\hat{\gamma}$ and $\hat{\lambda}$ is the MLEs of $\gamma$ and $\lambda$.

The approximate variance-covariance $(\mathrm{V}-\mathrm{C})$ matrix of the MLEs of $\gamma$ and $\lambda$ is

$$
I(\gamma, \lambda)=\left[\begin{array}{cc}
-E\left(\frac{\partial^{2} \mathscr{L}}{\partial \gamma^{2}}\right) & -E\left(\frac{\partial^{2} \mathscr{L}}{\partial \gamma \partial \lambda}\right) \\
-E\left(\frac{\partial^{2} \mathscr{L}}{\partial \lambda \partial \gamma}\right) & -E\left(\frac{\partial^{2} \mathscr{L}}{\partial \lambda^{2}}\right)
\end{array}\right],
$$

where

$$
\begin{aligned}
\frac{\partial^{2} \mathscr{L}}{\partial \gamma^{2}} & =\frac{-r}{\gamma^{2}}-\lambda \sum_{i=1}^{r} x_{i}^{-\gamma}\left(\ln x_{i}\right)^{2}-\sum_{j=1}^{m} \frac{\lambda R_{j} C_{j} \ln T_{q_{j}}}{A_{j}^{2}}, \\
\frac{\partial^{2} \mathscr{L}}{\partial \lambda^{2}} & =\frac{-r}{\lambda^{2}}-\sum_{j=1}^{m} \frac{B_{j} R_{j}}{A_{j}^{2}}, \\
\frac{\partial^{2} \mathscr{L}}{\partial \gamma \partial \lambda} & =\sum_{i=1}^{r} x_{i}^{-\gamma} \ln x_{i}-\sum_{j=1}^{m} \frac{R_{j} C_{j}}{A_{j}^{2}},
\end{aligned}
$$

where $B_{j}=\left(\partial A_{j} / \partial \lambda\right)=\exp \left(\lambda / T_{q_{j}}^{\gamma}\right)$ and $C_{j}=\left(\partial A_{j} / \partial \gamma\right)=$ $\ln T_{q_{j}}\left[A_{j}-\lambda B_{j}\right]$. Paper [18] came to the conclusion that the approximation $\mathrm{V}-\mathrm{C}$ matrix might be constructed by substituting anticipated values with their MLEs. The estimated sample information matrix will now be generated as

$$
I(\hat{\gamma}, \hat{\lambda})=-\left[\begin{array}{cc}
\frac{\partial^{2} \mathscr{L}}{\partial \gamma^{2}} & \frac{\partial^{2} \mathscr{L}}{\partial \gamma \partial \lambda} \\
\frac{\partial^{2} \mathscr{L}}{\partial \lambda \partial \gamma} & \frac{\partial^{2} \mathscr{L}}{\partial \lambda^{2}}
\end{array}\right],
$$

and hence the approximate V-C matrix of $\hat{\gamma}$ and $\hat{\lambda}$ will be

$$
\left[\begin{array}{ll}
\sigma_{11} & \sigma_{12} \\
\sigma_{21} & \sigma_{22}
\end{array}\right]=-\left[\begin{array}{ll}
\frac{\partial^{2} \mathscr{L}}{\partial \gamma^{2}} & \frac{\partial^{2} \mathscr{L}}{\partial \gamma \partial \lambda} \\
\frac{\partial^{2} \mathscr{L}}{\partial \lambda \partial \gamma} & \frac{\partial^{2} \mathscr{L}}{\partial \lambda^{2}}
\end{array}\right]_{\gamma=\hat{\gamma}, \lambda=\hat{\lambda}}^{-1} .
$$

Focused on the empirical distribution of the MLLE of the parameters, CIs for the unknown parameters $\gamma$ and $\lambda$ will be computed. It is established from the empirical distribution of the MLLE of the parameters that

$$
(\hat{\gamma}, \hat{\lambda})-(\gamma, \lambda) \longrightarrow N_{2}\left(0, I^{-1}(\hat{\gamma}, \hat{\lambda})\right),
$$

where $N_{2}(\cdot)$ is bivariate normal distribution and $I(\cdot)$ is the the Fisher information matrix which is defined in equation (12).

Considering specific regularity constraints, the twosided $100(1-\alpha) \%, 0<\alpha<1$, ACIs for the unknown parameters $\gamma$ and $\lambda$ can be obtained as $\hat{\gamma} \pm Z_{\alpha / 2} \sqrt{\sigma_{11}}$ and $\widehat{\lambda} \pm Z_{\alpha / 2} \sqrt{\sigma_{22}}$, where $\sigma_{11}$ and $\sigma_{22}$ are the asymptotic Vs of the MLEs of $\gamma$ and $\lambda$, respectively; here $Z_{\alpha / 2}$ is the upper $\alpha / 2^{\text {th }}$ percentile of the standard normal distribution.

\section{Bayesian Estimation}

In this part, we will look at how to use Bayesian estimation to estimate the unknown parameters of an IW distribution using a PCTI method. The SEr loss function will be used for Bayesian parameter estimation. It is possible to use separate gamma priors for both parameters of the IW distribution $\gamma$ and $\lambda$ with pdfs

$$
\begin{aligned}
& \pi_{1}(\gamma) \propto \gamma^{a_{1}-1} \exp \left(-b_{1} \gamma\right), \gamma>0, a_{1}>0, b_{1}>0, \\
& \pi_{2}(\lambda) \propto \lambda^{a_{2}-1} \exp \left(-b_{2} \lambda\right), \lambda>0, a_{2}>0, b_{2}>0,
\end{aligned}
$$

The hyperparameters $a_{1}, b_{1}, a_{2}, b_{2}$ are used to represent past knowledge of the unknown parameters in this situation. The joint prior (JP) for $\gamma$ and $\lambda$ is as follows:

$$
\pi(\gamma, \lambda) \propto \gamma^{a_{1}-1} \lambda^{a_{2}-1} \exp \left(-b_{1} \gamma-b_{2} \lambda\right) .
$$

Hyperparameter elicitation: the informative priors (IPs) will be used to elicit the hyperparameters. The above IPs will indeed be deduced from the MLEs for $(\gamma, \lambda)$ by equating the $M$ and $V$ of $\left(\hat{\gamma}^{j}, \widehat{\lambda}^{j}\right)$ with both the $M$ and $V$ of the regarded priors (Gamma priors), where $j=1,2, \ldots, k$ and $k$ is the number of observations from the IW distribution that are 
available. Thus, on equating $M$ and $V$ of $\left(\widehat{\gamma}^{j}, \hat{\lambda}^{j}\right)$ with the $M$ and $V$ of gamma priors, we acquire (29)

$$
\begin{aligned}
& \frac{1}{k} \sum_{j=1}^{k} \hat{\gamma}^{j}=\frac{a_{1}}{b_{1}} \text { and } \frac{1}{k-1} \sum_{j=1}^{k}\left(\hat{\gamma}^{j}-\frac{1}{k} \sum_{j=1}^{k} \hat{\gamma}^{j}\right)^{2}=\frac{a_{1}}{b_{1}^{2}} \\
& \frac{1}{k} \sum_{j=1}^{k} \hat{\lambda}^{j}=\frac{a_{2}}{b_{2}} \text { and } \frac{1}{k-1} \sum_{j=1}^{k}\left(\hat{\lambda}^{j}-\frac{1}{k} \sum_{j=1}^{k} \hat{\lambda}^{j}\right)^{2}=\frac{a_{2}}{b_{2}^{2}} .
\end{aligned}
$$

The calculated hyperparameters may now be expressed as after solving the preceding two equations

$$
\begin{aligned}
& a_{1}=\frac{\left(1 / k \sum_{j=1}^{k} \hat{\gamma}^{j}\right)^{2}}{1 / k-1 \sum_{j=1}^{k}\left(\hat{\gamma}^{j}-1 / k \sum_{j=1}^{k} \hat{\gamma}^{j}\right)^{2}} \text { and } b_{1}=\frac{1 / k \sum_{j=1}^{k} \hat{\gamma}^{j}}{1 / k-1 \sum_{j=1}^{k}\left(\hat{\gamma}^{j}-1 / k \sum_{j=1}^{k} \hat{\gamma}^{j}\right)^{2}}, \\
& a_{2}=\frac{\left(1 / k \sum_{j=1}^{k} \hat{\lambda}^{j}\right)^{2}}{1 / k-1 \sum_{j=1}^{k}\left(\hat{\lambda}^{j}-1 / k \sum_{j=1}^{k} \hat{\lambda}^{j}\right)^{2}} \text { and } b_{2}=\frac{1 / k \sum_{j=1}^{k} \hat{\lambda}^{j}}{1 / k-1 \sum_{j=1}^{k}\left(\hat{\lambda}^{j}-1 / k \sum_{j=1}^{k} \hat{\lambda}^{j}\right)^{2}} . \\
& \pi(\gamma, \lambda \mid x)=\frac{\pi(\gamma, \lambda) L(\gamma, \lambda)}{\int_{0}^{\infty} \int_{0}^{\infty} \pi(\gamma, \lambda) L(\gamma, \lambda) d \gamma d \lambda} .
\end{aligned}
$$

According to the observed data, the appropriate posterior density (PD) $x=\left(x_{(1)}, x_{(2)}, \ldots, x_{(r)}\right)$ can indeed be expressed as

The PD function is denoted by the symbol

$$
\pi(\gamma, \lambda \mid x)=K^{-1}\left[\gamma^{r+a_{1}-1} \lambda^{r+a_{2}-1} \exp \left(-b_{1} \gamma-b_{2} \lambda\right) \prod_{i=1}^{r}\left(x_{i}^{-(\gamma+1)} \exp \left(\frac{-\lambda}{x_{i}^{\gamma}}\right)\right) \prod_{j=1}^{m}\left(1-\exp \left(\frac{-\lambda}{T_{q_{j}}^{\gamma}}\right)\right)^{R_{j}}\right],
$$

where

$$
K=\int_{0}^{\infty} \int_{0}^{\infty} \gamma^{r+a_{1}-1} \lambda^{r+a_{2}-1} \exp \left(-b_{1} \gamma-b_{2} \lambda\right) \prod_{i=1}^{r}\left(x_{i}^{-(\gamma+1)} \exp \left(\frac{-\lambda}{x_{i}^{\gamma}}\right)\right) \prod_{j=1}^{m}\left(1-\exp \left(\frac{-\lambda}{T_{q_{j}}^{\gamma}}\right)\right)^{R_{j}} d \gamma d \lambda
$$

As a result, the PD may be rewritten as follows:

$$
\pi(\gamma, \lambda \mid x) \propto \gamma^{r+a_{1}-1} \lambda^{r+a_{2}-1} \exp \left(-b_{1} \gamma-b_{2} \lambda\right) \prod_{i=1}^{r}\left(x_{i}^{-(\gamma+1)} \exp \left(\frac{-\lambda}{x_{i}^{\gamma}}\right)\right) \prod_{j=1}^{m}\left(1-\exp \left(\frac{-\lambda}{T_{q_{j}}^{\gamma}}\right)\right)^{R_{j}}
$$

Under the SEr, the Bayes estimator of any function, such as $g(\gamma, \lambda)$, is provided by

$$
\tilde{g}(\gamma, \lambda)=\int_{0}^{\infty} \int_{0}^{\infty} g(\gamma, \lambda) \pi(\gamma, \lambda \mid x) d \gamma d \lambda .
$$

Consequently, equation (24) cannot be calculated for general $g(\gamma, \lambda)$. As a result, we recommend using the most widely used approximate BEs of $\gamma$ and $\lambda$ MCMC.
3.1. Lindley's Approximation. Lindley proposed an approximation to compute the ratio of integrals of the form in equation (25) for the specified priors on $\gamma$ and $\lambda$ and under the SEr loss function. Consider the ratio $L(X)$

$$
L(X)=\frac{\int_{(\gamma, \lambda)} g(\gamma, \lambda) \exp [\mathscr{L}(\gamma, \lambda)+\rho(\gamma, \lambda)] d(\gamma, \lambda)}{\int_{(\gamma, \lambda)} \exp [\mathscr{L}(\gamma, \lambda)+\rho(\gamma, \lambda)] d(\gamma, \lambda)},
$$


where $g(\gamma, \lambda)$ is function of $\gamma$ and $\lambda$ only and $\mathscr{L}(\gamma, \lambda)$ is the $\log$-LL given in $(5)$ and $\rho(\gamma, \lambda)$ is the log-JP distribution. Using the approach developed by [19], the ratio $L(X)$ can be expressed as

$$
\begin{aligned}
& L(X)=\hat{g}(\gamma, \lambda)+\frac{1}{2}\left[\left(\widehat{g}_{\gamma \gamma}+2 \widehat{g}_{\gamma} \widehat{\rho}_{\gamma}\right) \widehat{\sigma}_{\gamma \gamma}+\left(\widehat{g}_{\lambda \gamma}+2 \widehat{g}_{\lambda} \widehat{\rho}_{\gamma}\right) \widehat{\sigma}_{\lambda \gamma}\right. \\
& \left.+\left(\widehat{g}_{\gamma \lambda}+2 \widehat{g}_{\gamma} \widehat{\rho}_{\lambda}\right) \widehat{\sigma}_{\gamma \lambda}+\left(\widehat{g}_{\lambda \lambda}+2 \widehat{g}_{\lambda} \widehat{\rho}_{\lambda}\right) \widehat{\sigma}_{\lambda \lambda}\right] \\
& +\frac{1}{2}\left[( \widehat { g } _ { \gamma } \widehat { \sigma } _ { \gamma \gamma } + \widehat { g } _ { \lambda } \widehat { \sigma } _ { \gamma \lambda } ) \left(\hat{\mathscr{L}}_{\gamma \gamma \gamma} \widehat{\sigma}_{\gamma \gamma}+\hat{\mathscr{L}}_{\gamma \lambda \gamma} \widehat{\sigma}_{\gamma \lambda}+\hat{\mathscr{L}}_{\lambda \gamma \gamma} \widehat{\sigma}_{\lambda \gamma}\right.\right. \\
& \left.+\hat{\mathscr{L}}_{\lambda \lambda \gamma} \widehat{\sigma}_{\lambda \lambda}\right)+\left(\hat{g}_{\gamma} \widehat{\sigma}_{\lambda \gamma}+\widehat{\mathscr{g}}_{\lambda} \widehat{\sigma}_{\lambda \lambda}\right)\left(\hat{\mathscr{L}}_{\lambda \gamma \gamma} \widehat{\sigma}_{\gamma \gamma}+\hat{\mathscr{L}}_{\gamma \lambda \lambda} \widehat{\sigma}_{\gamma \lambda} \hat{\mathscr{L}}_{\lambda \gamma \lambda}\right. \\
& \left.+\widehat{\sigma}_{\lambda \gamma}+\widehat{\mathscr{L}}_{\lambda \lambda \lambda} \widehat{\sigma}_{\lambda \lambda}\right)
\end{aligned}
$$

where

$$
\begin{gathered}
\hat{g}_{i}=\left.\frac{\partial g(\gamma, \lambda)}{\partial i}\right|_{\gamma=\hat{\gamma}, \lambda=\hat{\lambda}} \hat{g}_{i j}=\left.\frac{\partial^{2} g(\gamma, \lambda)}{\partial i \partial j}\right|_{\gamma=\hat{\gamma}, \lambda=\hat{\lambda}} \hat{\rho}_{i}=\left.\frac{\partial \rho(\gamma, \lambda)}{\partial i}\right|_{\gamma=\hat{\gamma}, \lambda=\hat{\lambda}}, \\
\hat{\mathscr{L}}_{i j}=\left.\frac{\partial^{2} \mathscr{L}(\gamma, \lambda)}{\partial i \partial j}\right|_{\gamma=\hat{\gamma}, \lambda=\hat{\lambda}} \hat{\mathscr{L}}_{i j k}=\left.\frac{\partial^{3} \mathscr{L}(\gamma, \lambda)}{\partial i \partial j \partial k}\right|_{\gamma=\hat{\gamma}, \lambda=\hat{\lambda}} \hat{\sigma}=-\left.\frac{1}{\hat{\mathscr{L}}}\right|_{\gamma=\hat{\gamma}, \lambda=\hat{\lambda}} .
\end{gathered}
$$

Partial derivatives of $\mathscr{L}$ in equations (7)-(11) are also

$$
\begin{aligned}
& \mathscr{L}_{\gamma \gamma \gamma}=\frac{2 r}{\gamma^{3}}+\lambda \sum_{i=1}^{r} x_{i}^{-\gamma}\left(\ln x_{i}\right)^{3}-\sum_{j=1}^{m} \lambda R_{j} \ln T_{q_{j}}\left(K_{j} A_{j}-2 C_{j}^{v}\right) A_{j}^{3}, \\
& \mathscr{L}_{\lambda \lambda \lambda}=\frac{2 r}{\lambda^{3}}-\sum_{j=1}^{m} R_{j}\left(D_{j} A_{j}-2 B_{j}^{2}\right) A_{j}^{3}, \\
& \mathscr{L}_{\gamma \lambda \gamma}=-\sum_{i=1}^{r} x_{i}^{-\gamma}\left(\ln x_{i}\right)^{2}-\sum_{j=1}^{m} R_{j}\left(K_{j} A_{j}-2 C_{j}^{2}\right) A_{j}^{3},
\end{aligned}
$$$$
\mathscr{L}_{\gamma \lambda \lambda}=-\sum_{j=1}^{m} \frac{E_{j} R_{j}\left(A_{j}-2 B_{j}\right)}{A_{j}^{3}},
$$

where $\quad D_{j}=\left(\partial B_{j} / \partial \lambda\right)=T_{q_{j}}^{-\gamma} B_{j}, \quad E_{j}=\left(\partial B_{j} / \partial \gamma\right)=$ $-\lambda T_{q_{j}}^{-\gamma} \ln T_{q_{j}} B_{j}, \quad F_{j}=\left(\partial C_{j} / \partial \lambda\right)=-\lambda D_{j} \ln T_{q_{j}}$, and $K_{j}=\left(\partial C_{j} / \partial \gamma\right)=\ln T_{q_{j}}\left[B_{j}-\lambda E_{j}\right]$.

The $\log$-JP is given as

$$
\begin{aligned}
\rho(\gamma, \lambda)= & \log [\pi(\gamma, \lambda)]=C+\left(a_{1}-1\right) \log \gamma \\
& +\left(a_{2}-1\right) \lambda-b_{1} \gamma-b_{2} \lambda .
\end{aligned}
$$

Thus, the partial derivatives of log-JP distribution are

$$
\begin{gathered}
\rho_{\gamma}=\frac{\partial \rho(\gamma, \lambda)}{\partial \gamma}=\frac{a_{1}-1}{\gamma}-b_{1}, \\
\rho_{\lambda}=\frac{\partial \rho(\gamma, \lambda)}{\partial \lambda}=\frac{a_{2}-1}{\lambda}-b_{2} .
\end{gathered}
$$

Under the SEr function, the BE of $\gamma$ is already provided

$$
\begin{aligned}
g(\gamma, \lambda)= & \gamma \Rightarrow g_{\gamma}=1, g_{\lambda}=g_{\gamma \gamma}=g_{\gamma \lambda}=g_{\lambda \gamma}=g_{\lambda \lambda} \\
& =0 \text { and } \mathscr{L}_{\gamma \lambda \lambda}=0 .
\end{aligned}
$$

By substituting equations (7)-(11), (18)-(33) in (26), the estimates of $\gamma$ and $\lambda$ can be written as $\widehat{\gamma}_{\text {Lindley }}$ and $\hat{\lambda}_{\text {Lindley }}$.

3.2. Metropolis-Hasting Algorithm. We need to specify IW model and beginning values for the unknown parameters $\gamma$ and $\lambda$ to run the MH method for the IW distribution. We explore a bivariate normal distribution for the proposal distribution, that is, $q\left(\left(\gamma^{\prime}, \lambda^{\prime}\right) \mid(\gamma, \lambda)\right)=N_{2}\left((\gamma, \lambda), S_{\gamma, \lambda}\right)$. We may get negative observations, which are undesirable, if $S \gamma, \lambda$ represents the variance-covariance matrix. We use the MLE for $\gamma$ and $\lambda$ to determine the starting values, that is, $\left(\gamma^{(0)}, \lambda^{(0)}\right)=(\hat{\gamma}, \hat{\lambda})$. The selection of $S_{\gamma, \lambda}$ is examined to be the asymptotic V-C matrix $I^{-1}(\hat{\gamma}, \hat{\lambda})$, where $I($.$) is the Fisher$ information matrix. It is worth mentioning that the choice of $S_{\gamma, \lambda}$ is critical in the $\mathrm{MH}$ Algorithm 1, since the acceptance rate is determined by it. The following steps are followed used by the $\mathrm{MH}$ method to draw a sample from the PD given by equation (24) supplied in the following manner.

$$
\begin{cases}\text { If } u \leq \beta & \operatorname{set} \theta^{(i)}=\theta^{\prime} \\ \text { otherwise } & \operatorname{set} \theta^{(i)}=\theta .\end{cases}
$$

Eventually, using the PD's random samples of size $M$, part of the initial samples can indeed be eliminated (burnin), and the remaining samples can be used to produce BEs. Extra precisely equation (24) can be estimated as

$$
\widetilde{\mathfrak{g}}_{\mathrm{MH}}(\gamma, \lambda)=\frac{1}{M-l_{B}} \sum_{i=l_{B}}^{M} g\left(\gamma_{i}, \lambda_{i}\right),
$$

where $l_{B}$ is the total number of burn-in samples.

3.3. Highest Posterior Density (HDP). We use the samples generated from the proposed $\mathrm{MH}$ method in the preceding subsection to create HPD intervals for the unknown parameters $\gamma$ and $\lambda$ of the IW distribution under PCTI. Consider the following scenario: $\gamma^{(\delta)}$ and $\lambda^{(\delta)}$ are the $\delta$ th $Q$ of $\gamma$ and $\lambda$, respectively, that is,

$$
\left(\gamma^{(\delta)}, \lambda^{(\delta)}\right)=\inf \{(\gamma, \lambda): \Pi((\gamma, \lambda) \mid x) \geq \delta\}
$$

where $0<\delta<1$ and $\Pi(\cdot)$ is the posterior distribution function of $\gamma$ and $\lambda$. It is worth noting that, for specific $\gamma^{*}$ and $\lambda^{*}$, a simulation accurate estimator of $\pi((\gamma, \lambda) \mid x)$ may be calculated as

$$
\Pi\left(\left(\gamma^{*}, \lambda^{*}\right) \mid x\right)=\frac{1}{M-l_{B}} \sum_{i=l_{B}}^{M} I_{(\gamma, \lambda) \leq\left(\gamma^{*}, \lambda^{*}\right)} .
$$

Here $I_{(\gamma, \lambda) \leq\left(\gamma^{*}, \lambda^{*}\right)}$ is the indicator function. Then the appropriate estimate is computed as 
First Step Put initial value of $\theta$ as $\theta^{(0)}=(\hat{\gamma}, \hat{\lambda})$.

Second Step For $i=1,2, \ldots, M$ the necessary phases should be repeated: 2.1: Set $\theta=\theta^{(i-1)}$.

2.2: Create a new value for the candidate parameter $\delta$ from $N_{2}\left(\ln \theta, S_{\theta}\right)$.

2.3: Set $\theta^{\prime}=\exp (\delta)$.

2.4: Compute $\beta=\left(\pi\left(\theta^{\prime} \mid x\right) / \pi\left(\theta^{\prime} x\right)\right)$, where $\pi(\cdot)$ is the PD.

2.5: Construct a sample $u$ from the uniform $U(0,1)$ model

2.6: Accept or deny the new request according to $\theta^{\prime}$

Algorithm 1: Algorithm of MCMC.

$$
\widehat{\Pi}\left(\left(\gamma^{*}, \lambda^{*}\right) \mid x\right)= \begin{cases}0 & \text { if }\left(\gamma^{*}, \lambda^{*}\right)<\left(\gamma_{\left(l_{B}\right)}, \lambda_{\left(l_{B}\right)}\right) \\ \sum_{j=l_{B}}^{i} \omega_{j} & \text { if }\left(\gamma_{(i)}, \lambda_{(i)}\right)<\left(\gamma^{*}, \lambda^{*}\right)<\left(\gamma_{(i+1)}, \lambda_{(i+1)}\right), \\ 1 & \text { if }\left(\gamma^{*}, \lambda^{*}\right)>\left(\gamma_{(M)}, \lambda_{(M)}\right)\end{cases}
$$

where $\omega_{j}=\left(1 / M-l_{B}\right)$ and $\left(\gamma_{(j)}, \lambda_{(j)}\right)$ are the ordered values of $\left(\gamma_{j}, \lambda_{j}\right)$. Now, for $i=l_{B}, \ldots, M,\left(\gamma^{(\delta)}, \lambda^{(\delta)}\right)$ can be approximated by

$$
\left(\tilde{\gamma}^{(\delta)}, \tilde{\lambda}^{(\delta)}\right)= \begin{cases}\left(\gamma_{\left(l_{B}\right)}, \lambda_{(l-B)}\right) & \text { if } \delta=0 \\ \left(\gamma_{(i)}, \lambda_{(i)}\right) & \text { if } \sum_{j=l_{B}}^{i-1} \omega_{j}<\delta<\sum_{j=l_{B}}^{i} \omega_{j} .\end{cases}
$$

Let us now compute a $100(1-\delta) \%$ HPD credible interval for $\gamma$ and $\lambda$

$$
\begin{aligned}
\operatorname{HPD}_{j}^{\gamma} & =\left(\tilde{\gamma}^{(j / M)}, \tilde{\gamma}^{(j+(1-\delta) M / M)}\right) \text { and } \mathrm{HPD}_{j}^{\lambda} \\
& =\left(\tilde{\lambda}^{(j / M)}, \tilde{\lambda}^{(j+(1-\delta) M / M)}\right),
\end{aligned}
$$

for $j=l_{B}, \ldots,[\delta M]$; here $[a]$ represents the biggest integer that is less than or equal to $a$. Then select $\mathrm{HPD}_{j^{*}}$ from among all $\mathrm{HPD}_{j}^{\prime} s$ with the shortest width.

\section{Simulation Study and Real Data Application}

4.1. Simulation Study. In this part, we use a Monte Carlo simulation study to evaluate the performance of estimation approaches, namely, MLLE and Bayesian estimation using MCMC and Lindley's approximation, for the IW distribution using a PCTI scheme. We create 1000 data sets from the IW distribution for the MLEs under the next assumptions:

(1) Two initial values are $\operatorname{IW}(\gamma=2, \lambda=1.5)$ and $\operatorname{IW}(\gamma=1, \lambda=2)$

(2) Sample sizes are $n=25,50,100$

(3) Number of stages of PCTI is $m=3,4,5$

(4) Censoring time $T_{q_{i}}$ is calculated on the basis for the identified $q_{j}^{\text {th }}$ Qs from $\operatorname{IW}(2,1.5)$ and $\operatorname{IW}(1,2)$ :

(a) At $m=3$ and $q_{j}=(10 \%, 40 \%, 70 \%)$ (b) At $m=4$ and $q_{j}=(10 \%, 30 \%, 50 \%, 70 \%)$

(c) At $m=5$ and $q_{j}=(10 \%, 25 \%, 40 \%, 55 \%, 70 \%)$

(5) Omitted items $R_{j}$ are proposed based on a fraction of sample size $n$ where the number of removed items in each stage of censoring can be computed from

$$
R_{j}=\left\lceil\frac{f \% n}{m-1}\right\rceil, f=0 \%, 25 \%, 50 \% .
$$

Thus, the proposed schemes of removing items can be

Scheme I $(f=0 \%): R_{1}=R_{2}=\cdots=R_{m-1}=0$,

Scheme II $\quad(f=25 \%): \quad R_{1}=R_{2}=\cdots=R_{m-1}=$ $\lceil 25 \% n / m-1\rceil$

Scheme III $\quad(f=50 \%): \quad R_{1}=R_{2}=\cdots=R_{m-1}=$ $\lceil 50 \% n / m-1\rceil$,

When $R_{m}=n-\left(\sum_{j=1}^{m-1} R_{j}+r\right)$ and $r$ is the number of failure items and $\rceil\rceil$ is the ceiling function. It is indicated that scheme I represents Type-I censoring scheme where $R_{m}=n-r$.

We construct MLEs and related $95 \%$ asymptotic CIs premised on the data that is generated. When constructing MLEs, the initial estimate values are assumed to be the same as the real parameter values.

Under the informative prior (IP), we calculate BEs using the $\mathrm{MH}$ algorithm for Bayesian estimation. Thus, we have the following.

(i) As previous samples for the gamma prior, we produce 1000 complete samples of size 60 each from the $\operatorname{IW}(2,1.5)$ and $\operatorname{IW}(1,2)$ distributions, and then obtain the hyperparameter values accordingly: $a_{1}=22.74, b_{1}=14.20, a_{2}=9.65, b_{2}=4.29$.

To compute the desired estimations, the aforementioned IP values are entered in. We use the MLEs as starting guess values, as well as the related V-C matrix $S_{\theta}$ of $(\ln (\widehat{\lambda}), \ln (\widehat{\alpha}))$ when using the MH algorithm. Finally, we eliminated 2000 
burn-in samples from the total of 10,000 samples generated by the PD and then used [20] approach to get BEs and HPD interval estimations.

Tables 1-6 show the average estimates for both techniques. In addition, the first row displays average estimates (AVEs) and interval estimates (IEs), while the second row displays related mean square errors (MSErs) and average lengths (ALs) with coverage probabilities (CPrs). It can be seen from the table of values that, depending on MSErs, larger values of $n$ result in better estimates. It is also worth noting that MLEs outperform Lindley BEs and that the performance of BEs produced using MCMC outperforms Bayes estimates obtained via LiA. It is also worth noting that MCMC's ALs and related CPrs for HPD intervals outperform LiA. Furthermore, when the units are eliminated at an early stage in scheme II and scheme III, MSErs and ALs of related interval estimations are typically smaller.

4.2. Real Data Application. Two real data set are investigated for illustration and also to examine the statistical performance of the MLEs and BEs for the IW distribution under different PCTI censoring schemes.

4.2.1. Data Set I. The accompanying basic data set corresponds to an unfiltered data set. The data collection includes 34 observations of vinyl chloride data from [21], which indicates cleanup gradient ground-water monitoring wells in $\mathrm{mg} / \mathrm{L}$.

We begin by determining if the IW distribution is appropriate for studying this data set. To assess the quality of fit, we provide the MLEs of the parameters as well as the value of the Kolmogorov-Smirnov (KS) test statistic. The estimated KS and $p$-value for the IW distribution are 0.1161 and 0.7496 , respectively, where $\hat{\gamma}=0.8431$ and $\widehat{\lambda}=0.7121$, which indicate that this distribution can be considered as an adequate model for the given data set.

From the raw data, one may construct, for example, three PCTI censored samples with $m=3,4,5$ stages at time censoring $T_{q_{j}}$ matching to the chosen $q_{j}^{\text {th }}$, Qs, where $j=1, \ldots, m$. The sequences of deleted elements are presented for three fractions of eliminating $f=0 \%, 25 \%, 50 \%$ of total sample size $(n=34)$. Table 7 describes these patterns of removal process.

Here, $\left(1^{* 4}, 0\right)$ indicates, for example, that the censoring scheme used is $(1,1,1,1,0) \backslash$ enleadertwodots

In Table 8 , the MLEs of the parameters $\gamma$ and $\lambda$, as well as their related standard error and ACI at suggested schemes for PCTI samples in the provided real data set, have been computed.

Additionally, BEs were computed using the $\mathrm{MH}$ algorithm under the noninformative prior, i.e., $a 1=b 1=a 2=b 2=0$. It is said while using the MH method to generate samples from the posterior distribution, starting values of $(\gamma, \lambda)$ are regarded as $\left(\gamma^{(0)}, \lambda^{(0)}\right)=(\hat{\gamma}, \widehat{\lambda})$ and $\hat{\gamma}, \hat{\lambda}$ are the MLEs of the parameters $\gamma, \lambda$. Afterwards, 2000 burnin samples were eliminated from the overall 10000 samples generated by the $\mathrm{PD}$, and BEs and HPD intervals were produced. BEs based on LiA have been calculated based on the MLEs.

The convergence of MCMC estimate is in the case of PCTI scheme III for the data set I where the percentage of removal is $f=50 \%$. The BEs utilizing MCMC are converged through three subgraphs, as shown in Figure 2: scatter plot, histogram, and cumulative mean of the 10000 estimates.

4.2.2. Data Set II. A real data set of the carbonation depth of pier of a reinforced concrete girder bridge was analyzed under progressive Type-I censoring scheme [22]. The data set represents 27 measurements which are

2.0, 2.1, 2.2, 2.3, 2.3, 2.3, 2.4, 2.5, 2.6, 2.7, 2.8, 2.9, 3.0, 3.2, $3.2,3.3,3.3,3.3,3.4,3.4,3.4,3.5,3.5,3.6,3.7,3.8,3.9$.

First, we check whether the IW distribution is suitable for analyzing this data set. Also, we provide the MLEs of the parameters as well as the value of the Kolmogorov-Smirnov (KS) test statistic. The estimated KS and $p$-value for the IW distribution are 0.2074 and 0.1957 , respectively, where $\widehat{\gamma}=$ 5.2000 and $\hat{\lambda}=157.8003$, which indicate that this distribution can be considered as an adequate model for the given data set.

Two different PCTI censored samples are assumed for the given data set with $m=3,5$ stages at time censoring $T_{q_{j}}$ matching to the chosen $q_{j}^{t h}$, Qs, where $j=1, \ldots, m$. Table 9 describes the patterns of removing elements for three fractions of eliminating $f=0 \%, 25 \%, 50 \%$ of total sample size $(n=27)$.

In Table 10, the MLEs of the parameters $\gamma$ and $\lambda$, as well as their related ACI estimations and ACI at suggested schemes for PCTI samples in the provided real data set, have been computed.

As in data set I, BEs were computed using the $\mathrm{MH}$ algorithm under the noninformative prior. The starting values of $(\gamma, \lambda)$ are regarded as $\left(\gamma^{(0)}, \lambda^{(0)}\right)=(\hat{\gamma}, \hat{\lambda})$. Finally, 2000 burn-in samples were eliminated from the overall 10000 samples generated by the PD, and BEs and HPD intervals were produced. BEs based on LiA have been calculated based on the MLEs as a prior estimates.

Figure 3 illustrates the convergence of MCMC estimate in the case of PCTI scheme II for the real data set II where the percentage of removal is $f=50 \%$. The BEs utilizing MCMC are converged through three subgraphs, as shown in scatter plot, histogram, and cumulative mean of the 10000 estimates.

\section{Summary and Conclusion}

We investigated the topic of IW distribution estimation and prediction under PCTI from both classical and Bayesian perspectives in this work. For the unknown parameters of the IW distribution, we calculated maximum likelihood estimates and associated asymptotic confidence intervals. Then, using informative priors, we produced Bayes estimates and the related HPD interval estimates. In addition, when an informative prior is taken into account, a discussion of how to pick the values of hyperparameters in Bayesian estimation is examined based on historical samples. The simulation 
TABLe 1: Numerical results of AVEs, ACIs, MSErs, ALs, and CPrs (in \%) for $\gamma=2$ and $\lambda=1.5$ under number of stages $m=3$.

\begin{tabular}{|c|c|c|c|c|c|c|c|}
\hline \multirow{2}{*}{$f \%$} & \multirow{2}{*}{$n$} & \multirow{2}{*}{ Parm. } & \multicolumn{2}{|c|}{ MLLE } & \multicolumn{2}{|c|}{ Bayesian: MCMC } & \multirow{2}{*}{$\begin{array}{c}\text { Bayesian: Lindley } \\
\text { AVE MSEr }\end{array}$} \\
\hline & & & AVE MSEr & ACI AL/CPr & AVE MSEr & HPD AL/CPr & \\
\hline \multirow{12}{*}{0} & \multirow{4}{*}{25} & \multirow{2}{*}{$\gamma$} & 2.102 & $(1.344,2.861)$ & 2.078 & $(1.348,2.827)$ & 2.062 \\
\hline & & & 0.159 & $1.517 / 96.60$ & 0.155 & $1.479 / 96.60$ & 0.278 \\
\hline & & \multirow{2}{*}{$\lambda$} & 1.590 & $(0.952,2.228)$ & 1.579 & $(1.003,2.329)$ & 1.504 \\
\hline & & & 0.148 & $1.276 / 94.10$ & 0.142 & $1.326 / 96.10$ & 0.387 \\
\hline & \multirow{8}{*}{100} & \multirow{2}{*}{$\gamma$} & 2.053 & $(1.536,2.570)$ & 2.040 & $(1.536,2.584)$ & 2.042 \\
\hline & & & 0.078 & $1.034 / 95.30$ & 0.077 & $1.048 / 96.10$ & 0.102 \\
\hline & & \multirow{2}{*}{$\lambda$} & 1.541 & $(1.109,1.972)$ & 1.535 & $(1.137,2.022)$ & 1.522 \\
\hline & & & 0.054 & $0.863 / 95.40$ & 0.053 & $0.885 / 97.00$ & 0.090 \\
\hline & & \multirow{2}{*}{$\gamma$} & 2.029 & $(1.669,2.389)$ & 2.023 & $(1.655,2.363)$ & 2.026 \\
\hline & & & 0.034 & $0.720 / 96.50$ & 0.034 & $0.708 / 96.20$ & 0.040 \\
\hline & & \multirow{2}{*}{$\lambda$} & 1.513 & $(1.214,1.811)$ & 1.510 & $(1.207,1.807)$ & 1.507 \\
\hline & & & 0.024 & $0.597 / 96.60$ & 0.024 & $0.600 / 96.80$ & 0.032 \\
\hline \multirow{12}{*}{25} & \multirow{4}{*}{25} & \multirow[b]{2}{*}{$\gamma$} & 2.149 & $(1.328,2.970)$ & 2.123 & $(1.383,3.074)$ & 2.105 \\
\hline & & & 0.216 & $1.642 / 95.50$ & 0.211 & $1.691 / 97.30$ & 0.367 \\
\hline & & \multirow{2}{*}{$\lambda$} & 1.586 & $(0.937,2.235)$ & 1.577 & $(1.005,2.320)$ & 1.442 \\
\hline & & & 0.140 & $1.298 / 95.10$ & 0.134 & $1.315 / 97.00$ & 0.691 \\
\hline & \multirow{8}{*}{100} & \multirow[b]{2}{*}{$\gamma$} & 2.065 & $(1.509,2.621)$ & 2.051 & $(1.572,2.679)$ & 2.053 \\
\hline & & & 0.085 & $1.112 / 96.00$ & 0.084 & $1.107 / 97.90$ & 0.118 \\
\hline & & \multirow{2}{*}{$\lambda$} & 1.545 & $(1.103,1.987)$ & 1.541 & $(1.159,2.027)$ & 1.523 \\
\hline & & & 0.057 & $0.884 / 95.20$ & 0.056 & $0.868 / 97.00$ & 0.104 \\
\hline & & \multirow[b]{2}{*}{$\gamma$} & 2.028 & $(1.647,2.408)$ & 2.021 & $(1.648,2.414)$ & 2.023 \\
\hline & & & 0.039 & $0.761 / 96.40$ & 0.039 & $0.766 / 96.80$ & 0.047 \\
\hline & & \multirow{2}{*}{$\lambda$} & 1.522 & $(1.217,1.828)$ & 1.520 & $(1.192,1.833)$ & 1.517 \\
\hline & & & 0.027 & $0.611 / 95.90$ & 0.026 & $0.641 / 96.20$ & 0.037 \\
\hline \multirow{12}{*}{50} & \multirow{4}{*}{25} & & 2.167 & $(1.274,3.059)$ & 2.136 & $(1.274,3.055)$ & 2.091 \\
\hline & & $\gamma$ & 0.266 & $1.785 / 95.70$ & 0.256 & $1.780 / 96.00$ & 0.471 \\
\hline & & , & 1.578 & $(0.914,2.243)$ & 1.573 & $(0.922,2.322)$ & 1.298 \\
\hline & & 1 & 0.153 & $1.328 / 94.30$ & 0.149 & $1.399 / 96.20$ & 2.160 \\
\hline & & & 2.071 & $(1.481,2.660)$ & 2.055 & $(1.506,2.650)$ & 2.054 \\
\hline & 50 & $\gamma$ & 0.097 & $1.179 / 96.80$ & 0.095 & $1.144 / 97.10$ & 0.136 \\
\hline & 50 & & 1.541 & $(1.092,1.990)$ & 1.538 & $(1.112,2.013)$ & 1.505 \\
\hline & & $\lambda$ & 0.057 & $0.898 / 96.10$ & 0.056 & $0.901 / 96.80$ & 0.137 \\
\hline & & & 2.047 & $(1.642,2.452)$ & 2.040 & $(1.630,2.450)$ & 2.044 \\
\hline & 100 & $\gamma$ & 0.046 & $0.810 / 96.10$ & 0.046 & $0.820 / 96.50$ & 0.055 \\
\hline & 100 & & 1.518 & $(1.208,1.828)$ & 1.516 & $(1.206,1.825)$ & 1.510 \\
\hline & & $\lambda$ & 0.026 & $0.620 / 96.80$ & 0.026 & $0.619 / 96.80$ & 0.039 \\
\hline
\end{tabular}

Note: Parm.: parameter, AV: average, and ACI: asymptotic confidence interval.

TABle 2: Numerical results of AVEs, ACIs, MSErs, ALs, and CPrs (in \%) for $\gamma=2$ and $\lambda=1.5$ under number of stages $m=4$.

\begin{tabular}{|c|c|c|c|c|c|c|c|}
\hline \multirow{2}{*}{$f \%$} & \multirow{2}{*}{$n$} & \multirow{2}{*}{ Parm. } & \multicolumn{2}{|c|}{ MLLE } & \multicolumn{2}{|c|}{ Bayesian: MCMC } & \multirow{2}{*}{$\begin{array}{c}\text { Bayesian: Lindley } \\
\text { AVE MSEr }\end{array}$} \\
\hline & & & AVE MSEr & ACI AL/CPr & AVE MSEr & HPD AL/CPr & \\
\hline \multirow{12}{*}{0} & \multirow{4}{*}{25} & \multirow{2}{*}{$\gamma$} & 2.110 & $(1.349,2.871)$ & 2.086 & $(1.315,2.841)$ & 2.107 \\
\hline & & & 0.168 & $1.522 / 96.10$ & 0.164 & $1.526 / 96.20$ & 0.313 \\
\hline & & \multirow{2}{*}{$\lambda$} & 1.605 & $(0.960,2.249)$ & 1.593 & $(0.906,2.295)$ & 1.497 \\
\hline & & & 0.153 & $1.289 / 93.60$ & 0.148 & $1.389 / 95.30$ & 2.667 \\
\hline & \multirow{8}{*}{100} & \multirow[b]{2}{*}{$\gamma$} & 2.057 & $(1.539,2.576)$ & 2.045 & $(1.516,2.563)$ & 2.048 \\
\hline & & & 0.077 & $1.036 / 96.00$ & 0.076 & $1.047 / 96.00$ & 0.101 \\
\hline & & \multirow{2}{*}{$\lambda$} & 1.536 & $(1.106,1.966)$ & 1.530 & $(1.100,1.974)$ & 1.515 \\
\hline & & & 0.055 & $0.860 / 95.60$ & 0.053 & $0.874 / 95.90$ & 0.091 \\
\hline & & \multirow[b]{2}{*}{$\gamma$} & 2.025 & $(1.666,2.385)$ & 2.019 & $(1.688,2.363)$ & 2.031 \\
\hline & & & 0.032 & $0.719 / 97.30$ & 0.032 & $0.675 / 97.00$ & 0.039 \\
\hline & & \multirow{2}{*}{$\lambda$} & 1.513 & $(1.215,1.812)$ & 1.510 & $(1.243,1.823)$ & 1.501 \\
\hline & & & 0.023 & $0.596 / 96.90$ & 0.023 & $0.580 / 98.00$ & 0.033 \\
\hline
\end{tabular}


TABle 2: Continued.

\begin{tabular}{|c|c|c|c|c|c|c|c|}
\hline \multirow{2}{*}{$f \%$} & \multirow{2}{*}{$n$} & \multirow{2}{*}{ Parm. } & \multicolumn{2}{|c|}{ MLLE } & \multicolumn{2}{|c|}{ Bayesian: MCMC } & \multirow{2}{*}{$\begin{array}{c}\text { Bayesian: Lindley } \\
\text { AVE MSEr }\end{array}$} \\
\hline & & & AVE MSEr & ACI AL/CPr & AVE MSEr & HPD AL/CPr & \\
\hline \multirow{12}{*}{25} & \multirow{4}{*}{25} & \multirow[b]{2}{*}{$\gamma$} & 2.158 & $(1.308,3.007)$ & 2.127 & $(1.267,3.025)$ & 2.102 \\
\hline & & & 0.245 & $1.699 / 95.10$ & 0.237 & $1.758 / 96.00$ & 0.427 \\
\hline & & \multirow{2}{*}{$\lambda$} & 1.586 & $(0.931,2.242)$ & 1.578 & $(0.917,2.249)$ & 1.385 \\
\hline & & & 0.169 & $1.311 / 95.50$ & 0.169 & $1.331 / 96.10$ & 1.224 \\
\hline & \multirow{8}{*}{100} & \multirow[b]{2}{*}{$\gamma$} & 2.083 & $(1.520,2.645)$ & 2.069 & $(1.566,2.702)$ & 2.072 \\
\hline & & & 0.096 & $1.125 / 95.80$ & 0.094 & $1.136 / 97.70$ & 0.131 \\
\hline & & \multirow{2}{*}{$\lambda$} & 1.552 & $(1.109,1.995)$ & 1.548 & $(1.125,2.068)$ & 1.529 \\
\hline & & & 0.061 & $0.886 / 94.70$ & 0.059 & $0.943 / 97.10$ & 0.113 \\
\hline & & \multirow[t]{2}{*}{$\gamma$} & 2.032 & $(1.650,2.413)$ & 2.025 & $(1.607,2.409)$ & 2.033 \\
\hline & & & 0.042 & $0.763 / 96.00$ & 0.042 & $0.802 / 96.30$ & 0.046 \\
\hline & & \multirow{2}{*}{$\lambda$} & 1.517 & $(1.213,1.821)$ & 1.515 & $(1.218,1.810)$ & 1.523 \\
\hline & & & 0.028 & $0.608 / 96.60$ & 0.027 & $0.592 / 96.30$ & 0.037 \\
\hline \multirow{12}{*}{50} & \multirow{4}{*}{25} & \multirow[b]{2}{*}{$\gamma$} & 2.175 & $(1.271,3.078)$ & 2.141 & $(1.369,2.995)$ & 2.108 \\
\hline & & & 0.239 & $1.807 / 96.60$ & 0.227 & $1.626 / 96.30$ & 0.425 \\
\hline & & \multirow{2}{*}{$\lambda$} & 1.576 & $(0.917,2.234)$ & 1.571 & $(0.928,2.283)$ & 1.298 \\
\hline & & & 0.140 & $1.317 / 94.80$ & 0.136 & $1.354 / 96.20$ & 1.758 \\
\hline & \multirow{8}{*}{100} & \multirow[b]{2}{*}{$\gamma$} & 2.071 & $(1.481,2.659)$ & 2.054 & $(1.518,2.643)$ & 2.055 \\
\hline & & & 0.090 & $1.178 / 96.70$ & 0.088 & $1.125 / 96.60$ & 0.128 \\
\hline & & \multirow{2}{*}{$\lambda$} & 1.527 & $(1.085,1.970)$ & 1.524 & $(1.143,1.965)$ & 1.491 \\
\hline & & & 0.053 & $0.884 / 96.80$ & 0.052 & $0.821 / 97.00$ & 0.120 \\
\hline & & \multirow[b]{2}{*}{$\gamma$} & 2.030 & $(1.622,2.437)$ & 2.022 & $(1.604,2.408)$ & 2.031 \\
\hline & & & 0.047 & $0.815 / 97.00$ & 0.047 & $0.804 / 96.50$ & 0.056 \\
\hline & & \multirow{2}{*}{$\lambda$} & 1.522 & $(1.212,1.833)$ & 1.521 & $(1.222,1.864)$ & 1.519 \\
\hline & & & 0.029 & $0.621 / 95.80$ & 0.028 & $0.642 / 97.20$ & 0.039 \\
\hline
\end{tabular}

Note: Parm.: parameter, AV: average, and ACI: asymptotic confidence interval.

TABle 3: Numerical results of AVEs, ACIs, MSErs, ALs, and CPrs (in \%) for $\gamma=2$ and $\lambda=1.5$ under number of stages $m=5$.

\begin{tabular}{|c|c|c|c|c|c|c|c|}
\hline \multirow{2}{*}{$f \%$} & \multirow{2}{*}{$n$} & \multirow{2}{*}{ Parm. } & \multicolumn{2}{|c|}{ MLLE } & \multicolumn{2}{|c|}{ Bayesian: MCMC } & \multirow{2}{*}{$\begin{array}{c}\text { Bayesian: Lindley } \\
\text { AVE MSEr }\end{array}$} \\
\hline & & & AVE MSEr & $\mathrm{ACI} \mathrm{AL} / \mathrm{CPr}$ & AVE MSEr & HPD AL/CPr & \\
\hline \multirow{12}{*}{0} & \multirow{4}{*}{25} & \multirow[b]{2}{*}{$\gamma$} & 2.110 & $(1.352,2.869)$ & 2.086 & $(1.412,2.891)$ & 2.072 \\
\hline & & & 0.158 & $1.517 / 96.30$ & 0.154 & $1.479 / 97.30$ & 0.265 \\
\hline & & \multirow{2}{*}{$\lambda$} & 1.600 & $(0.958,2.241)$ & 1.588 & $(0.946,2.310)$ & 1.501 \\
\hline & & & 0.151 & $1.283 / 94.40$ & 0.144 & $1.364 / 95.90$ & 0.575 \\
\hline & \multirow{8}{*}{100} & \multirow[b]{2}{*}{$\gamma$} & 2.070 & $(1.550,2.590)$ & 2.058 & $(1.560,2.586)$ & 2.062 \\
\hline & & & 0.079 & $1.040 / 96.20$ & 0.077 & $1.026 / 96.70$ & 0.104 \\
\hline & & \multirow{2}{*}{$\lambda$} & 1.529 & $(1.101,1.956)$ & 1.522 & $(1.141,1.993)$ & 1.505 \\
\hline & & & 0.051 & $0.855 / 96.20$ & 0.049 & $0.852 / 97.90$ & 0.092 \\
\hline & & \multirow[b]{2}{*}{$\gamma$} & 2.024 & $(1.664,2.383)$ & 2.017 & $(1.698,2.415)$ & 2.020 \\
\hline & & & 0.034 & $0.719 / 96.10$ & 0.033 & $0.717 / 97.50$ & 0.039 \\
\hline & & \multirow{2}{*}{$\lambda$} & 1.521 & $(1.221,1.821)$ & 1.518 & $(1.217,1.827)$ & 1.516 \\
\hline & & & 0.025 & $0.600 / 95.80$ & 0.025 & $0.610 / 96.60$ & 0.032 \\
\hline \multirow{12}{*}{25} & \multirow{4}{*}{25} & \multirow{2}{*}{$\gamma$} & 2.156 & $(1.275,3.038)$ & 2.124 & $(1.331,3.074)$ & 2.099 \\
\hline & & & 0.228 & $1.763 / 95.50$ & 0.218 & $1.743 / 96.50$ & 0.412 \\
\hline & & \multirow{2}{*}{$\lambda$} & 1.596 & $(0.930,2.261)$ & 1.590 & $(0.931,2.268)$ & 1.412 \\
\hline & & & 0.206 & $1.331 / 94.70$ & 0.211 & $1.337 / 95.60$ & 0.936 \\
\hline & \multirow{8}{*}{100} & \multirow[b]{2}{*}{$\gamma$} & 2.070 & $(1.498,2.642)$ & 2.055 & $(1.541,2.668)$ & 2.057 \\
\hline & & & 0.093 & $1.144 / 96.10$ & 0.090 & $1.127 / 97.00$ & 0.127 \\
\hline & & \multirow{2}{*}{$\lambda$} & 1.542 & $(1.100,1.983)$ & 1.537 & $(1.105,1.977)$ & 1.512 \\
\hline & & & 0.055 & $0.883 / 96.60$ & 0.054 & $0.872 / 96.70$ & 0.119 \\
\hline & & \multirow[b]{2}{*}{$\gamma$} & 2.034 & $(1.645,2.423)$ & 2.026 & $(1.662,2.427)$ & 2.030 \\
\hline & & & 0.040 & $0.778 / 96.30$ & 0.040 & $0.765 / 96.80$ & 0.047 \\
\hline & & \multirow{2}{*}{$\lambda$} & 1.523 & $(1.217,1.828)$ & 1.521 & $(1.245,1.840)$ & 1.517 \\
\hline & & & 0.025 & $0.611 / 97.10$ & 0.024 & $0.595 / 97.70$ & 0.035 \\
\hline
\end{tabular}


TABle 3: Continued.

\begin{tabular}{|c|c|c|c|c|c|c|c|}
\hline \multirow{2}{*}{$f \%$} & \multirow{2}{*}{$n$} & \multirow{2}{*}{ Parm. } & \multicolumn{2}{|c|}{ MLLE } & \multicolumn{2}{|c|}{ Bayesian: MCMC } & \multirow{2}{*}{$\begin{array}{c}\text { Bayesian: Lindley } \\
\text { AVE MSEr }\end{array}$} \\
\hline & & & AVE MSEr & $\mathrm{ACI}$ AL/CPr & AVE MSEr & HPD AL/CPr & \\
\hline \multirow{12}{*}{50} & \multirow{4}{*}{25} & \multirow[b]{2}{*}{$\gamma$} & 2.178 & $(1.217,3.139)$ & 2.138 & $(1.311,3.250)$ & 2.076 \\
\hline & & & 0.309 & $1.922 / 94.40$ & 0.295 & $1.939 / 97.00$ & 0.575 \\
\hline & & \multirow{2}{*}{$\lambda$} & 1.594 & $(0.915,2.272)$ & 1.590 & $(0.876,2.263)$ & 1.242 \\
\hline & & & 0.193 & $1.357 / 95.60$ & 0.190 & $1.387 / 95.70$ & 3.589 \\
\hline & \multirow{8}{*}{100} & \multirow[b]{2}{*}{$\gamma$} & 2.101 & $(1.477,2.724)$ & 2.082 & $(1.504,2.775)$ & 2.084 \\
\hline & & & 0.119 & $1.247 / 95.70$ & 0.115 & $1.271 / 97.40$ & 0.166 \\
\hline & & \multirow{2}{*}{$\lambda$} & 1.541 & $(1.091,1.992)$ & 1.539 & $(1.041,2.019)$ & 1.493 \\
\hline & & & 0.064 & $0.901 / 94.80$ & 0.063 & $0.978 / 95.80$ & 0.175 \\
\hline & & \multirow[b]{2}{*}{$\gamma$} & 2.034 & $(1.615,2.452)$ & 2.025 & $(1.637,2.471)$ & 2.028 \\
\hline & & & 0.048 & $0.836 / 96.20$ & 0.047 & $0.833 / 97.30$ & 0.058 \\
\hline & & \multirow{2}{*}{$\lambda$} & 1.516 & $(1.206,1.827)$ & 1.515 & $(1.228,1.851)$ & 1.506 \\
\hline & & & 0.027 & $0.621 / 96.10$ & 0.027 & $0.622 / 97.20$ & 0.043 \\
\hline
\end{tabular}

Note: Parm.: parameter, AV: average, and ACI: asymptotic confidence interval.

Table 4: Numerical results of AVEs, ACIs, MSErs, ALs, and CPrs (in \%) for $\gamma=1$ and $\lambda=2$ under number of stages $m=3$.

\begin{tabular}{|c|c|c|c|c|c|c|c|}
\hline \multirow{2}{*}{$f \%$} & \multirow{2}{*}{$n$} & \multirow{2}{*}{ Parm. } & \multicolumn{2}{|c|}{ MLLE } & \multicolumn{2}{|c|}{ Bayesian: MCMC } & \multirow{2}{*}{$\begin{array}{c}\text { Bayesian: Lindley } \\
\text { AVE MSEr }\end{array}$} \\
\hline & & & AVE MSEr & ACI AL/CPr & AVE MSEr & HPD AL/CPr & \\
\hline \multirow{12}{*}{0} & \multirow{4}{*}{25} & \multirow[b]{2}{*}{$\gamma$} & 1.072 & $(0.688,1.456)$ & 1.060 & $(0.644,1.455)$ & 1.013 \\
\hline & & & 0.051 & $0.768 / 95.20$ & 0.050 & $0.811 / 95.60$ & 0.323 \\
\hline & & \multirow{2}{*}{$\lambda$} & 2.166 & $(1.251,3.081)$ & 2.148 & $(1.285,3.286)$ & 2.155 \\
\hline & & & 0.359 & $1.830 / 93.80$ & 0.356 & $2.001 / 95.70$ & 0.365 \\
\hline & \multirow{8}{*}{100} & \multirow[b]{2}{*}{$\gamma$} & 1.037 & $(0.776,1.299)$ & 1.031 & $(0.764,1.288)$ & 1.023 \\
\hline & & & 0.021 & $0.523 / 96.20$ & 0.020 & $0.524 / 96.00$ & 0.062 \\
\hline & & \multirow{2}{*}{$\lambda$} & 2.086 & $(1.480,2.692)$ & 2.075 & $(1.502,2.736)$ & 2.082 \\
\hline & & & 0.131 & $1.212 / 94.60$ & 0.128 & $1.234 / 96.10$ & 0.132 \\
\hline & & \multirow[b]{2}{*}{$\gamma$} & 1.011 & $(0.830,1.190)$ & 1.007 & $(0.852,1.202)$ & 1.002 \\
\hline & & & 0.009 & $0.360 / 96.90$ & 0.008 & $0.350 / 98.20$ & 0.016 \\
\hline & & \multirow{2}{*}{$\lambda$} & 2.025 & $(1.615,2.436)$ & 2.021 & $(1.614,2.436)$ & 2.025 \\
\hline & & & 0.046 & $0.821 / 95.80$ & 0.046 & $0.822 / 96.10$ & 0.047 \\
\hline \multirow{12}{*}{25} & \multirow{4}{*}{25} & \multirow[b]{2}{*}{$\gamma$} & 1.074 & $(0.663,1.485)$ & 1.061 & $(0.692,1.483)$ & 1.011 \\
\hline & & & 0.052 & $0.822 / 96.10$ & 0.053 & $0.791 / 96.70$ & 0.386 \\
\hline & & \multirow{2}{*}{$\lambda$} & 2.156 & $(1.236,3.077)$ & 2.150 & $(1.387,3.158)$ & 2.142 \\
\hline & & & 0.356 & $1.841 / 95.10$ & 0.357 & $1.771 / 96.60$ & 0.363 \\
\hline & \multirow{8}{*}{100} & \multirow[b]{2}{*}{$\gamma$} & 1.042 & $(0.762,1.322)$ & 1.035 & $(0.760,1.310)$ & 1.025 \\
\hline & & & 0.022 & $0.560 / 96.90$ & 0.021 & $0.550 / 96.90$ & 0.079 \\
\hline & & \multirow{2}{*}{$\lambda$} & 2.090 & $(1.476,2.703)$ & 2.081 & $(1.491,2.813)$ & 2.085 \\
\hline & & & 0.130 & $1.227 / 94.30$ & 0.127 & $1.322 / 97.10$ & 0.133 \\
\hline & & \multirow[b]{2}{*}{$\gamma$} & 1.013 & $(0.820,1.199)$ & 1.006 & $(0.831,1.193)$ & 0.999 \\
\hline & & & 0.010 & $0.379 / 96.50$ & 0.009 & $0.362 / 96.60$ & 0.019 \\
\hline & & \multirow{2}{*}{$\lambda$} & 2.029 & $(1.615,2.443)$ & 2.024 & $(1.608,2.435)$ & 2.028 \\
\hline & & & 0.046 & $0.828 / 96.50$ & 0.045 & $0.827 / 96.60$ & 0.047 \\
\hline \multirow{12}{*}{50} & \multirow{4}{*}{25} & & 1.086 & $(0.637,1.535)$ & 1.071 & $(0.657,1.649)$ & 0.964 \\
\hline & & $\gamma$ & 0.071 & $0.898 / 94.10$ & 0.070 & $0.992 / 97.00$ & 0.756 \\
\hline & & & 2.153 & $(1.225,3.082)$ & 2.140 & $(1.316,3.179)$ & 2.132 \\
\hline & & $\lambda$ & 0.303 & $1.857 / 95.00$ & 0.301 & $1.863 / 96.20$ & 0.322 \\
\hline & & & 1.034 & $(0.739,1.329)$ & 1.026 & $(0.744,1.339)$ & 0.994 \\
\hline & & $\gamma$ & 0.025 & $0.590 / 95.80$ & 0.025 & $0.595 / 96.90$ & 0.122 \\
\hline & 50 & 1 & 2.078 & $(1.464,2.693)$ & 2.070 & $(1.446,2.738)$ & 2.072 \\
\hline & & $\lambda$ & 0.127 & $1.229 / 95.20$ & 0.124 & $1.292 / 96.30$ & 0.132 \\
\hline & & & 1.014 & $(0.813,1.215)$ & 1.010 & $(0.814,1.211)$ & 1.002 \\
\hline & & $\gamma$ & 0.010 & $0.402 / 96.90$ & 0.010 & $0.397 / 96.70$ & 0.024 \\
\hline & 100 & $\lambda$ & 2.036 & $(1.616,2.455)$ & 2.031 & $(1.594,2.455)$ & 2.034 \\
\hline & & $\lambda$ & 0.054 & $0.839 / 95.30$ & 0.053 & $0.861 / 95.40$ & 0.055 \\
\hline
\end{tabular}

Note: Parm.: parameter, AV average, and ACI asymptotic confidence interval. 
TABle 5: Numerical results of AVEs, ACIs, MSErs, ALs, and CPrs (in \%) for $\gamma=1$ and $\lambda=2$ under number of stages $m=4$.

\begin{tabular}{|c|c|c|c|c|c|c|c|}
\hline \multirow{2}{*}{$f \%$} & \multirow{2}{*}{$n$} & \multirow{2}{*}{ Parm. } & \multicolumn{2}{|c|}{ MLLE } & \multicolumn{2}{|c|}{ Bayesian: MCMC } & \multirow{2}{*}{$\begin{array}{c}\text { Bayesian: Lindley } \\
\text { AVE MSEr }\end{array}$} \\
\hline & & & AVE MSEr & ACI AL/CPr & AVE MSEr & HPD AL/CPr & \\
\hline \multirow{12}{*}{0} & \multirow{4}{*}{25} & \multirow[b]{2}{*}{$\gamma$} & 1.074 & $(0.690,1.458)$ & 1.061 & $(0.683,1.436)$ & 1.036 \\
\hline & & & 0.046 & $0.768 / 96.10$ & 0.044 & $0.753 / 95.80$ & 0.264 \\
\hline & & \multirow{2}{*}{$\lambda$} & 2.161 & $(1.251,3.071)$ & 2.141 & $(1.326,3.224)$ & 2.149 \\
\hline & & & 0.326 & $1.820 / 94.00$ & 0.317 & $1.898 / 96.20$ & 0.333 \\
\hline & \multirow{8}{*}{100} & \multirow[b]{2}{*}{$\gamma$} & 1.027 & $(0.768,1.285)$ & 1.020 & $(0.762,1.274)$ & 1.005 \\
\hline & & & 0.018 & $0.517 / 96.80$ & 0.018 & $0.512 / 96.70$ & 0.059 \\
\hline & & \multirow{2}{*}{$\lambda$} & 2.075 & $(1.473,2.676)$ & 2.064 & $(1.526,2.738)$ & 2.071 \\
\hline & & & 0.116 & $1.203 / 95.00$ & 0.113 & $1.212 / 96.50$ & 0.117 \\
\hline & & \multirow[b]{2}{*}{$\gamma$} & 1.016 & $(0.835,1.196)$ & 1.013 & $(0.835,1.182)$ & 1.010 \\
\hline & & & 0.008 & $0.361 / 96.60$ & 0.007 & $0.347 / 96.30$ & 0.015 \\
\hline & & \multirow{2}{*}{$\lambda$} & 2.027 & $(1.616,2.437)$ & 2.021 & $(1.633,2.465)$ & 2.025 \\
\hline & & & 0.048 & $0.821 / 95.60$ & 0.047 & $0.832 / 96.90$ & 0.048 \\
\hline \multirow{12}{*}{25} & \multirow{4}{*}{25} & \multirow[b]{2}{*}{$\gamma$} & 1.089 & $(0.661,1.516)$ & 1.074 & $(0.674,1.538)$ & 1.032 \\
\hline & & & 0.060 & $0.855 / 94.90$ & 0.057 & $0.864 / 96.10$ & 0.475 \\
\hline & & \multirow{2}{*}{$\lambda$} & 2.166 & $(1.241,3.091)$ & 2.150 & $(1.359,3.343)$ & 2.145 \\
\hline & & & 0.334 & $1.850 / 94.10$ & 0.328 & $1.984 / 97.10$ & 0.351 \\
\hline & \multirow{8}{*}{100} & \multirow[b]{2}{*}{$\gamma$} & 1.036 & $(0.756,1.316)$ & 1.029 & $(0.744,1.310)$ & 1.012 \\
\hline & & & 0.022 & $0.560 / 95.90$ & 0.021 & $0.566 / 96.40$ & 0.083 \\
\hline & & \multirow{2}{*}{$\lambda$} & 2.070 & $(1.465,2.675)$ & 2.060 & $(1.498,2.777)$ & 2.064 \\
\hline & & & 0.118 & $1.210 / 94.70$ & 0.115 & $1.279 / 96.60$ & 0.121 \\
\hline & & \multirow{2}{*}{$\gamma$} & 1.015 & $(0.824,1.206)$ & 1.011 & $(0.832,1.238)$ & 1.005 \\
\hline & & & 0.011 & $0.382 / 95.60$ & 0.010 & $0.406 / 97.60$ & 0.022 \\
\hline & & \multirow{2}{*}{$\lambda$} & 2.038 & $(1.622,2.455)$ & 2.034 & $(1.608,2.441)$ & 2.037 \\
\hline & & & 0.051 & $0.833 / 95.90$ & 0.050 & $0.833 / 96.00$ & 0.052 \\
\hline \multirow{12}{*}{50} & \multirow{4}{*}{25} & & 1.083 & $(0.630,1.536)$ & 1.066 & $(0.630,1.523)$ & 0.976 \\
\hline & & $\gamma$ & 0.066 & $0.906 / 95.60$ & 0.063 & $0.893 / 96.00$ & 0.741 \\
\hline & & $\lambda$ & 2.173 & $(1.232,3.113)$ & 2.159 & $(1.288,3.363)$ & 2.148 \\
\hline & & $\lambda$ & 0.383 & $1.881 / 93.70$ & 0.381 & $2.075 / 95.80$ & 0.396 \\
\hline & & & 1.040 & $(0.744,1.336)$ & 1.031 & $(0.742,1.348)$ & 1.010 \\
\hline & & $\gamma$ & 0.026 & $0.592 / 95.80$ & 0.025 & $0.606 / 97.20$ & 0.110 \\
\hline & 50 & $\lambda$ & 2.066 & $(1.459,2.673)$ & 2.057 & $(1.498,2.721)$ & 2.060 \\
\hline & & $\lambda$ & 0.110 & $1.214 / 95.00$ & 0.108 & $1.224 / 96.10$ & 0.115 \\
\hline & & & 1.019 & $(0.814,1.223)$ & 1.014 & $(0.806,1.216)$ & 1.009 \\
\hline & & $\gamma$ & 0.011 & $0.409 / 96.60$ & 0.012 & $0.410 / 96.60$ & 0.025 \\
\hline & 100 & $\lambda$ & 2.030 & $(1.613,2.447)$ & 2.025 & $(1.646,2.510)$ & 2.028 \\
\hline & & $\lambda$ & 0.051 & $0.834 / 95.10$ & 0.054 & $0.864 / 97.60$ & 0.053 \\
\hline
\end{tabular}

Note: Parm.: parameter, AV: average, and ACI: asymptotic confidence interval.

Table 6: Numerical results of AVEs, ACIs, MSErs, ALs, and CPrs (in \%) for $\gamma=1$ and $\lambda=2$ under number of stages $m=5$.

\begin{tabular}{|c|c|c|c|c|c|c|c|}
\hline \multirow{2}{*}{$f \%$} & \multirow{2}{*}{$n$} & \multirow{2}{*}{ Parm. } & \multicolumn{2}{|c|}{ MLLE } & \multicolumn{2}{|c|}{ Bayesian: MCMC } & \multirow{2}{*}{$\begin{array}{c}\text { Bayesian: Lindley } \\
\text { AVE MSEr }\end{array}$} \\
\hline & & & AVE MSEr & $\mathrm{ACI} \mathrm{AL} / \mathrm{CPr}$ & AVE MSEr & HPD AL/CPr & \\
\hline \multirow{12}{*}{0} & \multirow{4}{*}{25} & \multirow[b]{2}{*}{$\gamma$} & 1.054 & $(0.675,1.432)$ & 1.042 & $(0.695,1.435)$ & 0.952 \\
\hline & & & 0.042 & $0.757 / 95.90$ & 0.041 & $0.740 / 97.00$ & 0.836 \\
\hline & & \multirow{2}{*}{$\lambda$} & 2.170 & $(1.259,3.080)$ & 2.151 & $(1.409,3.194)$ & 2.164 \\
\hline & & & 0.271 & $1.821 / 94.60$ & 0.262 & $1.785 / 96.80$ & 0.280 \\
\hline & \multirow{8}{*}{100} & \multirow[b]{2}{*}{$\gamma$} & 1.027 & $(0.768,1.287)$ & 1.021 & $(0.760,1.295)$ & 1.005 \\
\hline & & & 0.019 & $0.519 / 95.30$ & 0.019 & $0.535 / 96.20$ & 0.061 \\
\hline & & \multirow{2}{*}{$\lambda$} & 2.072 & $(1.471,2.673)$ & 2.063 & $(1.492,2.710)$ & 2.070 \\
\hline & & & 0.117 & $1.202 / 94.60$ & 0.115 & $1.218 / 96.20$ & 0.120 \\
\hline & & \multirow[b]{2}{*}{$\gamma$} & 1.017 & $(0.836,1.197)$ & 1.014 & $(0.842,1.196)$ & 1.011 \\
\hline & & & 0.009 & $0.361 / 96.90$ & 0.008 & $0.353 / 97.20$ & 0.016 \\
\hline & & \multirow{2}{*}{$\lambda$} & 2.048 & $(1.632,2.464)$ & 2.043 & $(1.640,2.456)$ & 2.047 \\
\hline & & & 0.049 & $0.831 / 96.80$ & 0.048 & $0.815 / 96.80$ & 0.049 \\
\hline
\end{tabular}


Table 6: Continued.

\begin{tabular}{|c|c|c|c|c|c|c|c|}
\hline \multirow{2}{*}{$f \%$} & \multirow{2}{*}{$n$} & \multirow{2}{*}{ Parm. } & \multicolumn{2}{|c|}{ MLLE } & \multicolumn{2}{|c|}{ Bayesian: MCMC } & \multirow{2}{*}{$\begin{array}{c}\text { Bayesian: Lindley } \\
\text { AVE MSEr }\end{array}$} \\
\hline & & & AVE MSEr & ACI AL/CPr & AVE MSEr & HPD AL/CPr & \\
\hline \multirow{12}{*}{25} & \multirow{4}{*}{25} & \multirow[b]{2}{*}{$\gamma$} & 1.092 & $(0.648,1.537)$ & 1.077 & $(0.687,1.621)$ & 1.002 \\
\hline & & & 0.071 & $0.889 / 93.10$ & 0.068 & $0.934 / 97.00$ & 0.677 \\
\hline & & \multirow{2}{*}{$\lambda$} & 2.171 & $(1.240,3.103)$ & 2.157 & $(1.299,3.244)$ & 2.149 \\
\hline & & & 0.345 & $1.863 / 93.70$ & 0.342 & $1.945 / 96.10$ & 0.363 \\
\hline & \multirow{8}{*}{100} & \multirow{2}{*}{$\gamma$} & 1.041 & $(0.753,1.328)$ & 1.033 & $(0.757,1.357)$ & 1.018 \\
\hline & & & 0.024 & $0.575 / 95.50$ & 0.023 & $0.600 / 97.00$ & 0.093 \\
\hline & & \multirow{2}{*}{$\lambda$} & 2.089 & $(1.477,2.702)$ & 2.079 & $(1.472,2.729)$ & 2.085 \\
\hline & & & 0.117 & $1.225 / 95.30$ & 0.114 & $1.257 / 96.30$ & 0.120 \\
\hline & & \multirow{2}{*}{$\gamma$} & 1.019 & $(0.824,1.214)$ & 1.015 & $(0.831,1.207)$ & 1.012 \\
\hline & & & 0.010 & $0.390 / 96.80$ & 0.009 & $0.376 / 96.60$ & 0.020 \\
\hline & & \multirow{2}{*}{$\lambda$} & 2.036 & $(1.620,2.452)$ & 2.032 & $(1.617,2.486)$ & 2.035 \\
\hline & & & 0.051 & $0.832 / 95.50$ & 0.051 & $0.869 / 96.60$ & 0.052 \\
\hline \multirow{12}{*}{50} & \multirow{4}{*}{25} & \multirow[b]{2}{*}{$\gamma$} & 1.112 & $(0.622,1.602)$ & 1.092 & $(0.665,1.701)$ & 1.009 \\
\hline & & & 0.091 & $0.980 / 93.90$ & 0.085 & $1.036 / 96.80$ & 0.951 \\
\hline & & \multirow{2}{*}{$\lambda$} & 2.188 & $(1.233,3.144)$ & 2.177 & $(1.319,3.410)$ & 2.154 \\
\hline & & & 0.410 & $1.911 / 93.40$ & 0.415 & $2.091 / 96.50$ & 0.430 \\
\hline & \multirow{8}{*}{100} & \multirow[b]{2}{*}{$\gamma$} & 1.052 & $(0.740,1.365)$ & 1.043 & $(0.740,1.369)$ & 1.030 \\
\hline & & & 0.031 & $0.625 / 95.30$ & 0.030 & $0.629 / 95.90$ & 0.126 \\
\hline & & \multirow{2}{*}{$\lambda$} & 2.079 & $(1.466,2.693)$ & 2.071 & $(1.509,2.775)$ & 2.071 \\
\hline & & & 0.120 & $1.227 / 94.80$ & 0.118 & $1.266 / 96.60$ & 0.125 \\
\hline & & \multirow[b]{2}{*}{$\gamma$} & 1.024 & $(0.813,1.234)$ & 1.019 & $(0.797,1.231)$ & 1.014 \\
\hline & & & 0.013 & $0.421 / 96.30$ & 0.012 & $0.434 / 96.60$ & 0.030 \\
\hline & & \multirow{2}{*}{$\lambda$} & 2.043 & $(1.623,2.464)$ & 2.039 & $(1.612,2.480)$ & 2.041 \\
\hline & & & 0.056 & $0.841 / 95.30$ & 0.055 & $0.868 / 96.60$ & 0.057 \\
\hline
\end{tabular}

Note: Parm.: parameter, AV: average, and ACI: asymptotic confidence interval.

TABLE 7: Removal patterns of units under different censoring schemes for data set I.

\begin{tabular}{|c|c|c|c|c|}
\hline Scheme & $m$ & $q_{j}(\%)$ & $T_{q_{j}}$ & $R_{i}$ \\
\hline I & 3 & $(10,40,70)$ & $(0.26,0.90,2.31)$ & $\begin{array}{l}f_{0 \%}: R=\left(0^{* 2}, R_{m}\right) \\
f_{25 \%}: R=\left(3^{* 2}, R_{m}\right) \\
f_{50 \%}: R=\left(5^{* 2}, R_{m}\right)\end{array}$ \\
\hline II & 4 & $(10,30,50,70)$ & $(0.26,0.59,1.15,2.31)$ & $\begin{array}{l}f_{0 \%}: R=\left(0^{* 3}, R_{m}\right) \\
f_{25 \%}: R=\left(2^{* 3}, R_{m}\right) \\
f_{50 \%}: R=\left(4^{* 3}, R_{m}\right)\end{array}$ \\
\hline III & 5 & $(10,25,40,55,70)$ & $(0.26,0.50,0.90,1.251,2.31)$ & $\begin{array}{l}f_{0 \%}: R=\left(0^{* 4}, R_{m}\right) \\
f_{25 \%}: R=\left(2^{* 4}, R_{m}\right) \\
f_{50 \%}: R=\left(3^{* 4}, R_{m}\right)\end{array}$ \\
\hline
\end{tabular}

TAble 8: MLL, Bayesian, and St.E and ACI based on the PCTI under various censoring schemes for data set I.

\begin{tabular}{|c|c|c|c|c|c|c|c|c|c|}
\hline \multirow{2}{*}{ Sch. } & \multirow{2}{*}{$f \%$} & \multirow{2}{*}{ Parm. } & \multicolumn{3}{|c|}{ MLL } & \multicolumn{3}{|c|}{ MCMC } & \multirow{2}{*}{$\begin{array}{l}\text { Lindley } \\
\text { Estimate }\end{array}$} \\
\hline & & & Estimate & St.E & ACI & Estimate & St.E & HPD & \\
\hline \multirow{6}{*}{ I } & \multirow{2}{*}{0} & $\gamma$ & 0.4720 & 0.0952 & $(0.2854,1.1864)$ & 0.6417 & 0.0165 & $(0.4024,0.9145)$ & 0.7053 \\
\hline & & $\lambda$ & 1.7210 & 0.2727 & $(0.6586,2.2556)$ & 1.0312 & 0.0555 & $(0.5647,1.4825)$ & 1.7534 \\
\hline & \multirow{2}{*}{25} & $\gamma$ & 0.5186 & 0.1131 & $(0.2968,1.0013)$ & 0.6151 & 0.0216 & $(0.3353,0.8893)$ & 0.8423 \\
\hline & & $\lambda$ & 1.5560 & 0.2830 & $(0.7405,2.1107)$ & 1.0192 & 0.0671 & $(0.5144,1.5035)$ & 1.6146 \\
\hline & \multirow{2}{*}{50} & $\gamma$ & 0.4557 & 0.1157 & $(0.2289,1.0803)$ & 0.5234 & 0.0198 & $(0.2898,0.8476)$ & 0.9699 \\
\hline & & $\lambda$ & 1.7423 & 0.3377 & $(0.6825,2.4043)$ & 1.2496 & 0.0945 & $(0.6814,1.8303)$ & 1.8185 \\
\hline
\end{tabular}


TABLE 8: Continued.

\begin{tabular}{|c|c|c|c|c|c|c|c|c|c|}
\hline \multirow{2}{*}{ Sch. } & \multirow{2}{*}{$f \%$} & \multirow{2}{*}{ Parm. } & \multicolumn{3}{|c|}{ MLL } & \multicolumn{3}{|c|}{ MCMC } & \multirow{2}{*}{$\begin{array}{l}\text { Lindley } \\
\text { Estimate }\end{array}$} \\
\hline & & & Estimate & St.E & $\mathrm{ACI}$ & Estimate & St.E & HPD & \\
\hline \multirow{6}{*}{ II } & \multirow{2}{*}{0} & $\gamma$ & 0.5406 & 0.1072 & $(0.3304,0.9283)$ & 0.6486 & 0.0166 & $(0.4117,0.9216)$ & 0.7447 \\
\hline & & $\lambda$ & 1.4382 & 0.2601 & $(0.7507,1.9482)$ & 1.0257 & 0.0545 & $(0.5853,1.4701)$ & 1.4749 \\
\hline & \multirow{2}{*}{25} & $\gamma$ & 0.6203 & 0.1207 & $(0.3838,0.8324)$ & 0.7199 & 0.0203 & $(0.4393,0.9876)$ & 0.8450 \\
\hline & & $\lambda$ & 1.3309 & 0.2543 & $(0.8569,1.8294)$ & 0.9202 & 0.0493 & $(0.5122,1.3606)$ & 1.3860 \\
\hline & \multirow{2}{*}{50} & $\gamma$ & 0.5892 & 0.1442 & $(0.3065,0.7183)$ & 0.5459 & 0.0247 & $(0.2531,0.8646)$ & 0.9890 \\
\hline & & $\lambda$ & 1.3319 & 0.3130 & $(0.8720,1.9455)$ & 1.1908 & 0.0990 & $(0.6207,1.8487)$ & 1.4416 \\
\hline \multirow{6}{*}{ III } & \multirow{2}{*}{0} & $\gamma$ & 0.5406 & 0.1072 & $(0.3304,0.9283)$ & 0.6427 & 0.0176 & $(0.3972,0.9138)$ & 0.7527 \\
\hline & & $\dot{\lambda}$ & 1.4382 & 0.2601 & $(0.7507,1.9482)$ & 1.0185 & 0.0561 & $(0.5831,1.4811)$ & 1.3028 \\
\hline & \multirow{2}{*}{25} & $\gamma$ & 0.6217 & 0.1417 & $(0.3439,0.6541)$ & 0.6094 & 0.0211 & $(0.3299,0.8943)$ & 0.9460 \\
\hline & & $\lambda$ & 1.1831 & 0.2698 & $(0.8994,1.7120)$ & 1.0463 & 0.0623 & $(0.5815,1.5315)$ & 1.2913 \\
\hline & \multirow{2}{*}{50} & $\gamma$ & 0.6971 & 0.1528 & $(0.3976,0.5897)$ & 0.6977 & 0.0288 & $(0.3841,1.0246)$ & 1.0644 \\
\hline & & $\lambda$ & 1.1005 & 0.2606 & $(0.9966,1.6114)$ & 0.9170 & 0.0671 & $(0.4681,1.4697)$ & 1.2497 \\
\hline
\end{tabular}

Note: ACI: asymptotic confidence interval, Parm.: parameter, Schs.: scheme, and St.E: standard error.
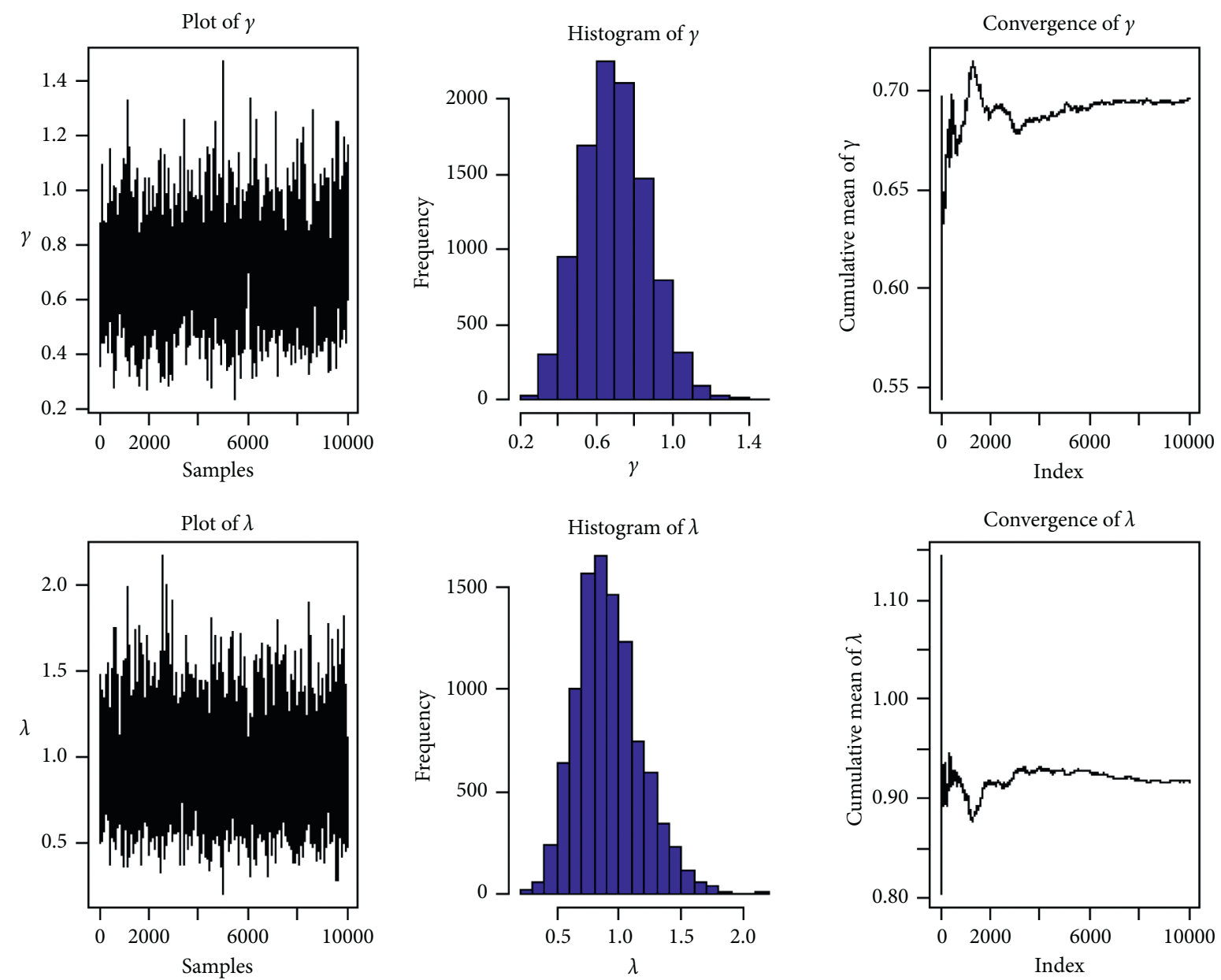

Figure 2: Convergence of MCMC estimates for $\gamma$ and $\lambda$ implementing the MH method for data set I under PCTI $m=5$ and $f=50 \%$.

outcomes demonstrate that MLEs informative Bayes estimates using Lindley approximation perform better than both MLEs and informative prior using Lindley approximation and that estimates under informative prior using MCMC perform better than both MLEs and informative prior using Lindley approximation. We used Bayesian estimation with the squared error loss function for future work, but other loss functions can also be used. In addition, the current approach may be extended to the construction of an optimum progressive censoring, as well as alternative 
TABLE 9: Removal patterns of units under different censoring schemes for data set II.

\begin{tabular}{|c|c|c|c|c|}
\hline Scheme & $m$ & $q_{j}(\%)$ & $T_{q_{j}}$ & $R_{i}$ \\
\hline I & 3 & $(10,40,70)$ & $(2.26,2.84,3.40)$ & $\begin{array}{l}f_{0 \%}: R=\left(0^{* 2}, R_{m}\right) \\
f_{25 \%}: R=\left(3^{* 2}, R_{m}\right) \\
f_{50 \%}: R=\left(5^{* 2}, R_{m}\right)\end{array}$ \\
\hline II & 5 & $(10,25,40,55,70)$ & $(2.26,2.45,2.84,3.23,3.40)$ & $\begin{array}{l}f_{0 \%}: R=\left(0^{* 4}, R_{m}\right) \\
f_{25 \%}: R=\left(2^{* 4}, R_{m}\right) \\
f_{50 \%}: R=\left(3^{* 4}, R_{m}\right)\end{array}$ \\
\hline
\end{tabular}

TABle 10: MLL, Bayesian, and St.E and ACI based on the PCTI under various censoring schemes for data set II.

\begin{tabular}{|c|c|c|c|c|c|c|c|c|c|}
\hline \multirow{2}{*}{ Sch. } & \multirow{2}{*}{$f \%$} & \multirow{2}{*}{ Parm. } & \multicolumn{3}{|c|}{ MLL } & \multicolumn{3}{|c|}{ MCMC } & \multirow{2}{*}{$\begin{array}{l}\text { Lindley } \\
\text { Estimate }\end{array}$} \\
\hline & & & Estimate & St.E & ACI & Estimate & St.E & HPD & \\
\hline \multirow{6}{*}{ I } & \multirow{2}{*}{0} & $\gamma$ & 2.9651 & 0.6477 & $(1.6130,5.6993)$ & 2.9660 & $9.34 \times 10^{(-5)}$ & $(2.9464,2.9853)$ & 2.9793 \\
\hline & & $\lambda$ & 27.0113 & 1.7130 & $(23.3172,33.3233)$ & 27.0115 & $1.01 \times 10^{(-4)}$ & $(26.9920,27.0312)$ & 27.0107 \\
\hline & \multirow{2}{*}{25} & $\gamma$ & 3.5567 & 0.8081 & $(2.0791,6.0904)$ & 3.5572 & $9.72 \times 10^{(-5)}$ & $(3.5382,3.5767)$ & 3.5741 \\
\hline & & $\lambda$ & 44.8461 & 3.3430 & $(24.0343,64.6019)$ & 44.8460 & $1.02 \times 10^{(-4)}$ & $(44.8270,44.8656)$ & 44.8457 \\
\hline & \multirow{2}{*}{50} & $\gamma$ & 3.2201 & 0.9133 & $(1.5435,7.6124)$ & 3.2204 & $9.93 \times 10^{(-5)}$ & $(3.2016,3.2404)$ & 3.2475 \\
\hline & & $\lambda$ & 35.1225 & 4.9036 & $(23.8966,46.6325)$ & 35.1224 & $1.06 \times 10^{(-4)}$ & $(35.1022,35.1414)$ & 35.1216 \\
\hline \multirow{6}{*}{ II } & \multirow{2}{*}{0} & $\gamma$ & 3.5062 & 0.7624 & $(3.0917,7.3792)$ & 3.5068 & $9.83 \times 10^{(-5)}$ & $(3.48812,3.5276)$ & 3.5222 \\
\hline & & $\lambda$ & 38.4492 & 2.7722 & $(23.9207,53.5191)$ & 38.4491 & $1.02 \times 10^{(-4)}$ & $(38.4290,38.4685)$ & 38.4487 \\
\hline & \multirow{2}{*}{25} & $\gamma$ & 4.6583 & 1.0922 & $(2.9813,10.7054)$ & 4.6585 & $9.88 \times 10^{(-5)}$ & $(4.6387,4.6772)$ & 4.6790 \\
\hline & & $\lambda$ & 59.4018 & 9.4702 & $(28.3353,78.0982)$ & 59.4020 & $1.06 \times 10^{(-4)}$ & $(29.3828,80.4223)$ & 59.4016 \\
\hline & \multirow{2}{*}{50} & $\gamma$ & 3.9068 & 1.1731 & $(2.0377,12.2449)$ & 3.9066 & $9.97 \times 10^{(-5)}$ & $(3.8868,3.9259)$ & 3.9306 \\
\hline & & $\lambda$ & 48.4916 & 13.8929 & $(18.7758,74.7383)$ & 48.4915 & $1.09 \times 10^{(-4)}$ & $(48.4720,48.5111)$ & 48.4911 \\
\hline
\end{tabular}

Note: ACI: asymptotic confidence interval, Parm.: parameter, Schs.: scheme, and St.E: standard error.
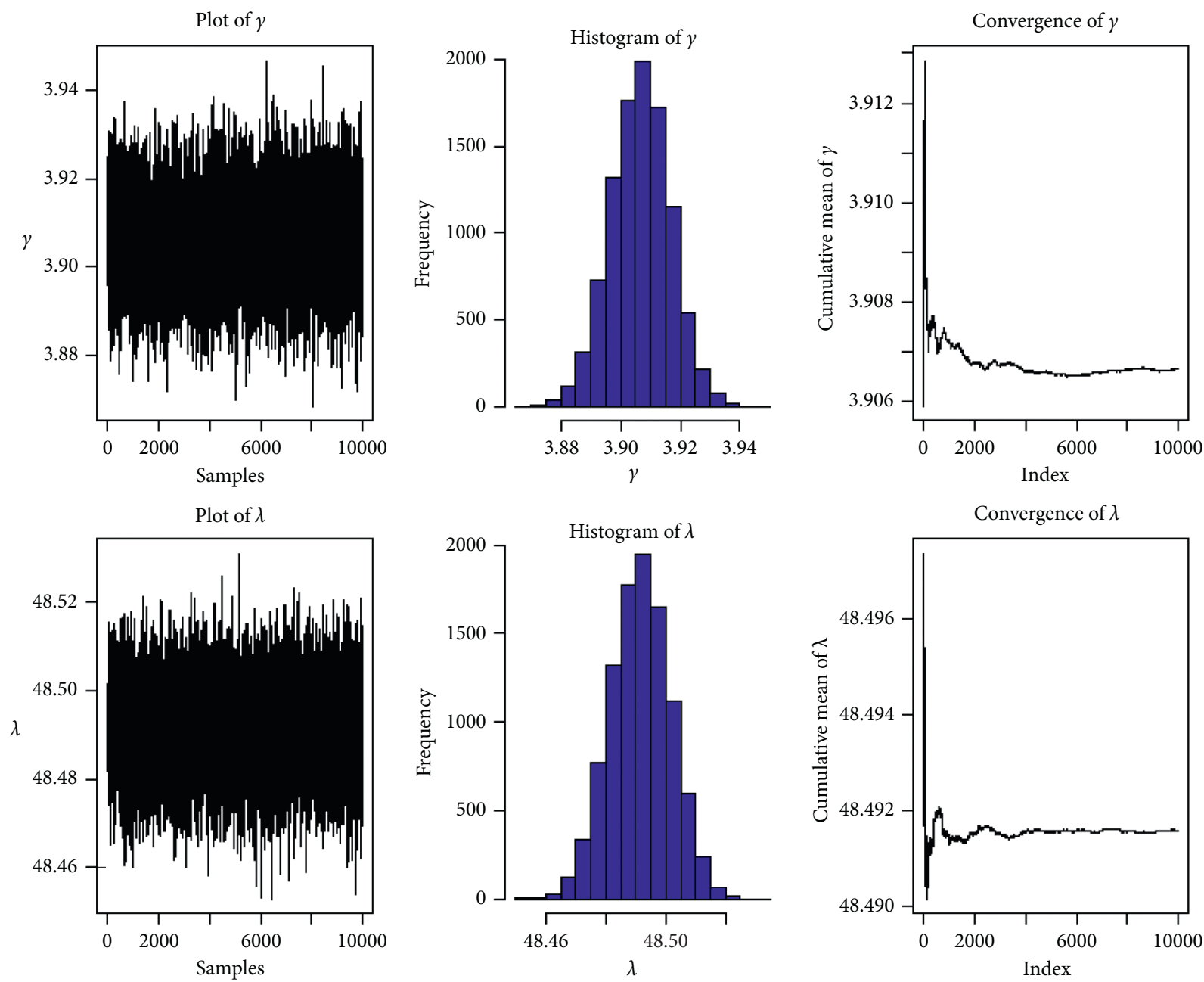

Figure 3: Convergence of MCMC estimates for $\gamma$ and $\lambda$ implementing the MH method for the data set II under PCTI $m=5$ and $f=50 \%$. 
censoring methods. Neutrosophic statistics can be an extended work on area of progressive censoring schemes under the assumed distribution and PCTTI.

\section{Data Availability}

Interested parties can reach out to the author in order to receive a numerical data set used to perform the research described in the study.

\section{Conflicts of Interest}

The authors declare that there are no conflicts of interest.

\section{Acknowledgments}

The Deanship of Scientific Research (DSR), King Abdul-Aziz University, Jeddah, 299, supported this work, under Grant no. KEP-PhD-75-130-42. The authors, 300 therefore, gratefully acknowledge the DSR technical and financial support.

\section{References}

[1] A. Z. Keller and A. R. R. Kamath, "Alternate reliability models for mechanical systems," ESA Reliab Maintainab, pp. 411-415, 1982.

[2] P. Erto, "Genesis, properties and identification of the inverse Weibull lifetime model," Statistics and Applications, vol. 1, pp. 117-128, 1989.

[3] R. Calabria and G. Pulcini, "Bayes 2-sample prediction for the inverse Weibull distribution," Communications in Statistics Theory and Methods, vol. 23, no. 6, pp. 1811-1824, 1994.

[4] R. M. Musleh and A. Helu, "Estimation of the inverse Weibull distribution based on progressively censored data: comparative study," Reliability Engineering \& System Safety, vol. 131, pp. 216-227, 2014.

[5] S. K. Singh, U. Singh, and D. Kumar, "Bayesian estimation of parameters of inverse Weibull distribution," Journal of Applied Statistics, vol. 40, no. 7, pp. 1597-1607, 2013.

[6] F. R. S. de Gusmão, E. M. M. Ortega, and G. M. Cordeiro, "The generalized inverse Weibull distribution," Statistical Papers, vol. 52, no. 3, pp. 591-619, 2011.

[7] I. Elbatal and H. Z. Muhammed, "Exponentiated generalized inverse Weibull distribution," Applied Mathematical Sciences, vol. 8, pp. 1597-1607, 2014.

[8] M. S. Khan, G. R. Pasha, and A. H. Pasha, "Theoretical analysis of inverse weibull distribution," WSEAS Transactions on Mathematics, vol. 7, pp. 30-38, 2008.

[9] P. Erto, "New practical Bayes estimators for the 2-parameter weibull distribution," IEEE Transactions on Reliability, vol. 31, pp. 194-197, 2009.

[10] K. S. Sultan, M. A. Ismail, and A. S. Al-Moisheer, "Mixture of two inverse Weibull distributions: properties and estimation," Computational Statistics \& Data Analysis, vol. 51, no. 11, pp. 5377-5387, 2007.

[11] N. Balakrishnan, D. Han, and G. Iliopoulos, "Exact inference for progressively type-I censored exponential failure data," Metrika, vol. 73, no. 3, pp. 335-358, 2011.

[12] U. Balasooriya and C.-K. Low, "Competing causes of failure and reliability tests for Weibull lifetimes under type I progressive censoring," IEEE Transactions on Reliability, vol. 53, no. 1, pp. 29-36, 2004.
[13] A. C. Cohen, "Progressively censored samples in life testing," Technometrics, vol. 5, no. 3, pp. 327-339, 1963.

[14] N. Balakrishnan and E. Cramer, The Art of Progressive Censoring: Applications to Reliability and Quality, Springer, New York, NY, USA, 2010.

[15] R. M. Mahmoud, H. Z. Muhammed, and A. R. El-Saeed, "Inference for generalized inverted exponential distribution under progressive Type-I censoring scheme in presence of competing risks model," Sankhya A: The Indian Journal of Statistics, vol. 134, p. 1, 2021, https://doi.org/10.1007/s13171020-00227-y.

[16] R. M. Mahmoud, H. Z. Muhammed, and A. R. El-Saeed, "Analysis of progressively Type-I censored data in competing risks models with generalized inverted exponential distribution," Journal of Statistics Applications \& Probability, vol. 9, pp. 109-117, 2020.

[17] M. R. Mahmoud, H. Z. Muhammed, A. R. El-Saeed, and A. D. Abdellatif, "Estimation of parameters of the GIE distribution under progressive Type-I censoring," Journal of Statistical Theory and Applications, vol. 20, no. 2, pp. 380-394, 2021.

[18] A. C. Cohen, "Maximum likelihood estimation in the weibull distribution based on complete and on censored samples," Technometrics, vol. 7, no. 4, pp. 579-588, 1965.

[19] D. V. Lindley, "Fiducial distributions and Bayes' theorem," Journal of the Royal Statistical Society: Series B, vol. 20, no. 1, pp. 102-107, 1958.

[20] M.-H. Chen and Q.-M. Shao, "Monte Carlo estimation of bayesian credible and HPD intervals," Journal of Computational \& Graphical Statistics, vol. 8, no. 1, pp. 69-92, 1999.

[21] D. K. Bhaumik, K. Kapur, and R. D. Gibbons, "Testing parameters of a gamma distribution for small samples," Technometrics, vol. 51, pp. 26-334, 2009.

[22] X. Guan, D. T. Niu, and J. B. Wang, "Carbonation service life prediction of coal boardwalks bridges based on durability testing," Journal of Xi'an University of Architecture and Technology, vol. 47, pp. 71-76, 2015. 